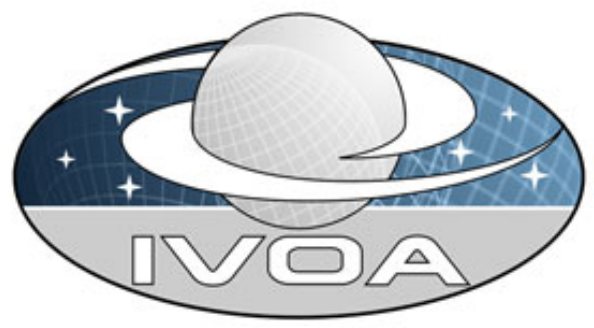

\title{
VODataService: a VOResource Schema Extension for Describing Collections and Services Version 1.1
}

IVOA Recommendation 02 December 2010

This version:

http://www.ivoa.net/Documents/VODataService/20101202

Latest version:

http://www.ivoa.net/Documents/VODataService

Previous versions:

PR: http://www.ivoa.net/Documents/VODataService/20100916

PR: http://www.ivoa.net/Documents/VODataService/20100914

PR: http://www.ivoa.net/Documents/VODataService/20100412

PR: http://www.ivoa.net/Documents/VODataService/20090903

WD: http://www.ivoa.net/Documents/WD/ReR/VODataService-20090508.html

\section{Authors:}

Raymond Plante, Editor

Aurélien Stébé

Kevin Benson

Patrick Dowler

Matthew Graham

Gretchen Greene

Paul Harrison

Gerard Lemson

Tony Linde

Guy Rixon

and the IVOA Registry Working Group.

\section{Abstract}

VODataService refers to an XML encoding standard for a specialized extension of the IVOA Resource Metadata that is useful for describing data collections and the services that access them. It is defined as an extension of the core resource metadata encoding standard known as VOResource [Plante et al. 2008] using XML Schema. The specialized resource types defined by the VODataService schema allow one to describe how the data 
underlying the resource cover the sky as well as cover frequency and time. This coverage description leverages heavily the Space-Time Coordinates (STC) standard schema [Rots 2007]. VODataService also enables detailed descriptions of tables that includes information useful to the discovery of tabular data. It is intended that the VODataService data types will be particularly useful in describing services that support standard IVOA service protocols.

\section{Status of this document}

This document has been produced by the IVOA Registry Working Group. It has been reviewed by IVOA Members and other interested parties, and has been endorsed by the IVOA Executive Committee as an IVOA Recommendation as 01 Oct 2010. It is a stable document and may be used as reference material or cited as a normative reference from another document. IVOA's role in making the Recommendation is to draw attention to the specification and to promote its widespread deployment. This enhances the functionality and interoperability inside the Astronomical Community.

A list of current IVOA Recommendations and other technical documents can be found at http://www.ivoa.net/Documents/.

\section{Acknowledgements}

This document has been developed with support from the National Science Foundation's Information Technology Research Program under Cooperative Agreement AST0122449 with The Johns Hopkins University, from the UK Particle Physics and Astronomy Research Council (PPARC), from the European Commission's (EC) Sixth Framework Programme via the Optical Infrared Coordination Network (OPTICON), and from EC's Seventh Framework Programme via its elnfrastructure Science Repositories initiative.

\section{Conformance-related definitions}

The words "MUST", "SHALL", "SHOULD", "MAY", "RECOMMENDED", and "OPTIONAL" (in upper or lower case) used in this document are to be interpreted as described in IETF standard, RFC 2119 [RFC 2119].

The Virtual Observatory (VO) is general term for a collection of federated resources that can be used to conduct astronomical research, education, and outreach. The International Virtual Observatory Alliance (IVOA) is a global collaboration of separately funded projects to develop standards and infrastructure that enable VO applications.

$X M L$ document validation is a software process that checks that an XML document is not only well-formed XML but also conforms to the syntax rules defined by the applicable schema. Typically, when the schema is defined by one or more XML Schema [schema] documents (see next section), validation refers to checking for conformance to the syntax described in those Schema documents. This document describes additional syntax constraints that cannot be enforced solely by the rules of XML Schema; thus, in this document, use of the term validation includes the extra checks that go beyond common Schema-aware parsers which ensure conformance with this document.

\section{Syntax Notation Using XML Schema}

The eXtensible Markup Language, or XML, is a document syntax for marking textual 
information with named tags and is defined by the World Wide Web Consortium (W3C) Recommendation, XML 1.0 [XML]. The set of XML tag names and the syntax rules for their use is referred to as the document schema. One way to formally define a schema for XML documents is using the W3C standard known as XML Schema [schema].

This document defines the VOResource schema using XML Schema. The full Schema document is listed in Appendix A. Parts of the schema appear within the main sections of this document; however, documentation nodes have been left out for the sake of brevity.

References to specific elements and types defined in the VOResource schema include the namespaces prefix, vr, as in vr:Resource (a type defined in the VOResource schema). References to specific elements and types defined in the VODataService extension schema include the namespaces prefix, vs, as in vs:Datacollection (a type defined in the VODataService schema). Use of the vs prefix in compliant instance documents is strongly recommended, particularly in the applications that involve IVOA Registries (see [RI], section 3.1.2). Elsewhere, the use is not required.

\title{
Contents
}

\author{
Abstract \\ Status of this document \\ Acknowledgments \\ Conformance-related definitions \\ Syntax Notation Using XML Schema \\ 1. Introduction \\ 1.1. The Role in the IVOA Architecture \\ 1.2. Purpose \\ 2. The VOResource Data Model \\ 2.1. The Schema Namespace and Location \\ 2.2. Summary of Metadata Concepts \\ 3. The VODataService Metadata \\ 3.1. Resource Type Extensions \\ 3.2. Coverage \\ 3.3. Tabular Data \\ 3.4. Interface Type Extension: ParamHTTP \\ 3.5. Data Parameters \\ Appendix A: the VODataService XML Schema \\ Appendix B: Compatibility Issues with VODataService 1.0 \\ Appendix C: Change History \\ References
}

\section{Introduction}

The VOResource standard [VOR] provides a means of encoding IVOA Resource Metadata [RM] in XML. VOResource uses XML Schema [schema] to define most of the XML syntax rules (while a few of the syntax rules are outside the scope of Schema). VOResource also describes mechanisms for creating extensions to the core VOResource metadata. This allows for the standardization of new metadata for describing specialized kinds of resources in a modular way without deprecating the core schema or other extensions. This document defines one such extension referred to as VODataService.

\subsection{The Role in the IVOA Architecture}


The IVOA Architecture [Arch] provides a high-level view of how IVOA standards work together to connect users and applications with providers of data and services, as depicted in the diagram in Fig. 1.

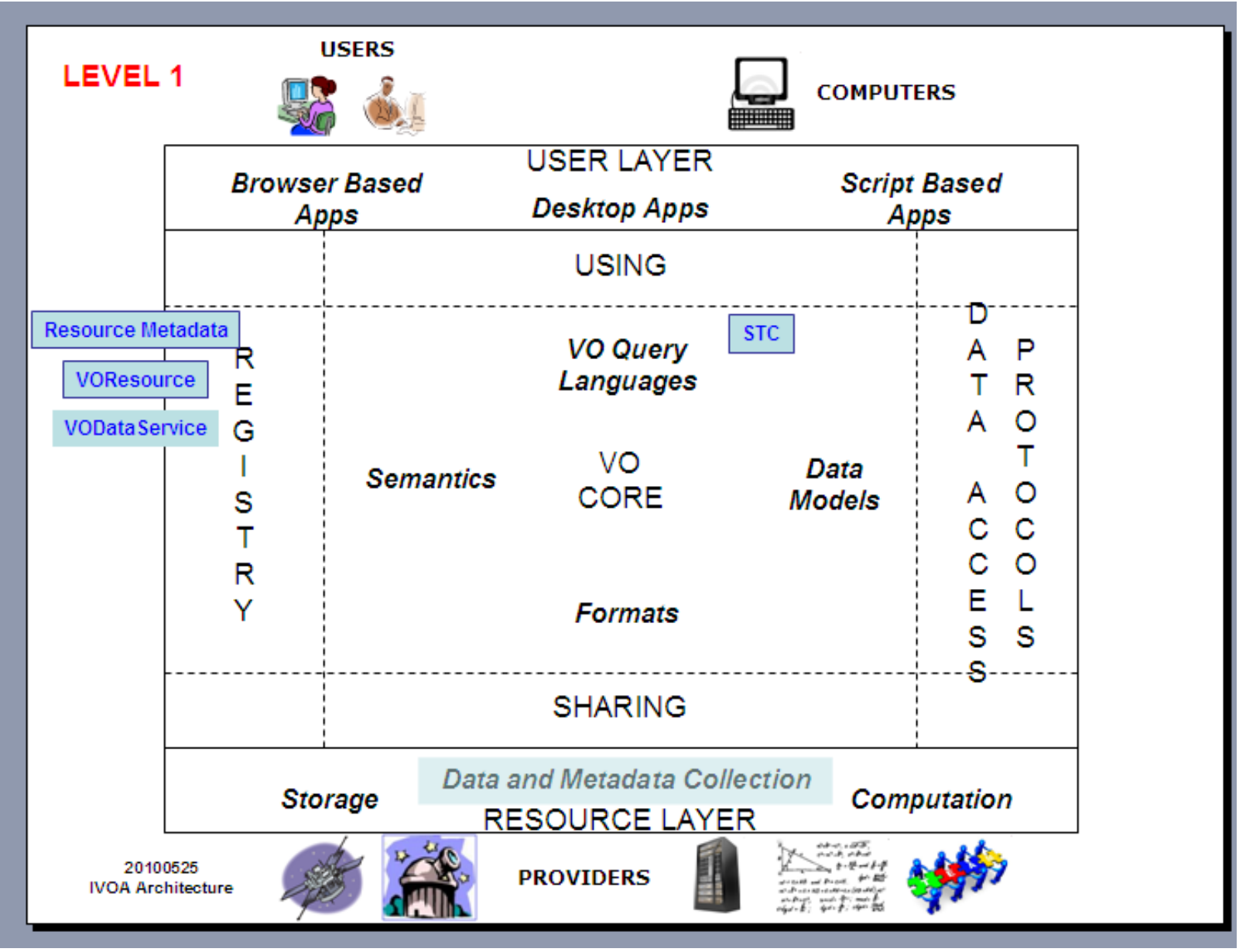

Figure 1. VODataService in the IVOA Architecture. The Registry enables applications in the User Layer to discover archives and services in the Resource Layer. The metadata and data model standards (in blue text and boxes) gives structure to the information that enables that discovery.

In this architecture, users can leverage a variety of tools (from the User Layer) to discover archives and services of interest (represented in the Resource Layer); registries provide the means for this discovery. A registry is a repository of descriptions of resources that can be searched based on the metadata in those descriptions. The Resource Metadata standard [RM] defines the core concepts used in the resource descriptions, and the VOResource standard [VOR] defines the XML format. As an extension of VOResource, the VODataService standard, defined in this document, specifically supports descriptions of data collections and services.

\subsection{Purpose}

The purpose of this extension is to define common XML Schema types--particularly new resource types--that are useful for describing data collections and services that access data. In particular, it allows one to describe the data's coverage: the parts of the sky with which the data are associated and the time and frequency ranges that were observed or modeled to create the data. It also allows one to describe tables in detail. In particular, 
one can describe each of the columns of a table--providing, for example, its name, type, UCD [UCD], and textual description. When this metadata is part of a resource description in a registry [ $\underline{\mathrm{VOR}}$ ], it becomes possible to discover tables that contain particular kinds of data.

It is intended that VODataService will be central to describing services that support standard IVOA data access layer protocols such as Simple Image Access [SIA] and Simple Cone Search [SCS]. While other VOResource extensions would define the protocol-specific metadata (encapsulated as a standard capability [VOR]), the general service resource description would share the common data concepts such as coverage and tabular data. Note, however, that a service described using the VODataService schema need not support any standard protocols. With the VODataService extension schema plus the core VOResource schema, it is possible to describe a custom service interface that accesses data.

As a legal extension of VOResource [VOR], the use of VODataService is subject to the rules and recommendations for XML [ $\underline{\mathrm{xml}}], \mathrm{XML}$ Schema [schema], and VOResource itself.

\section{The VODataService Data Model}

The VODataService extension in general enables the description of two types of resources: data collections and services that access data. Here's an example of a VOResource document (abbreviated for the purposes of illustration) that describes a service from the NASA Extragalactic Database (NED) that provides measured redshifts for a given object.

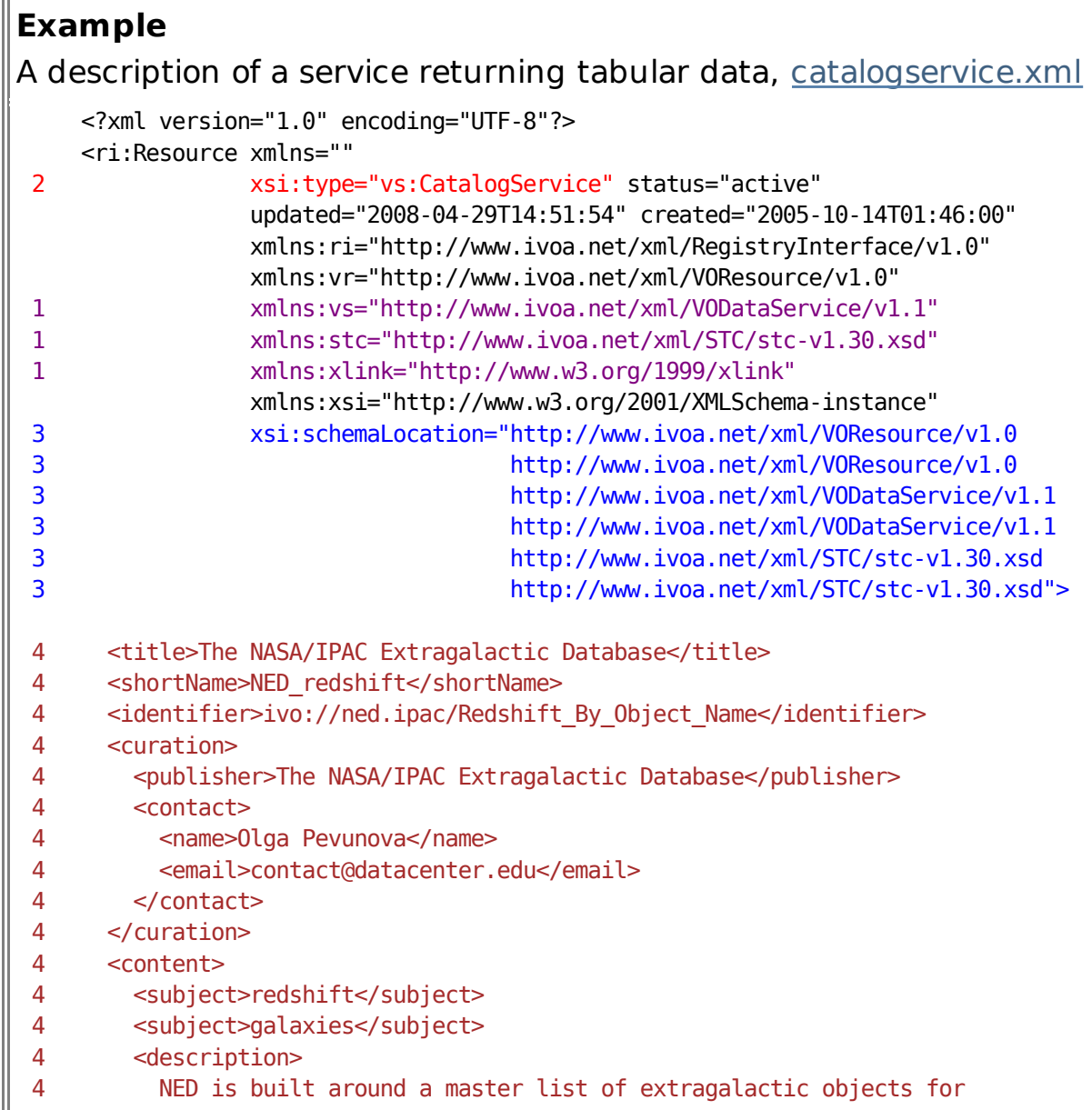




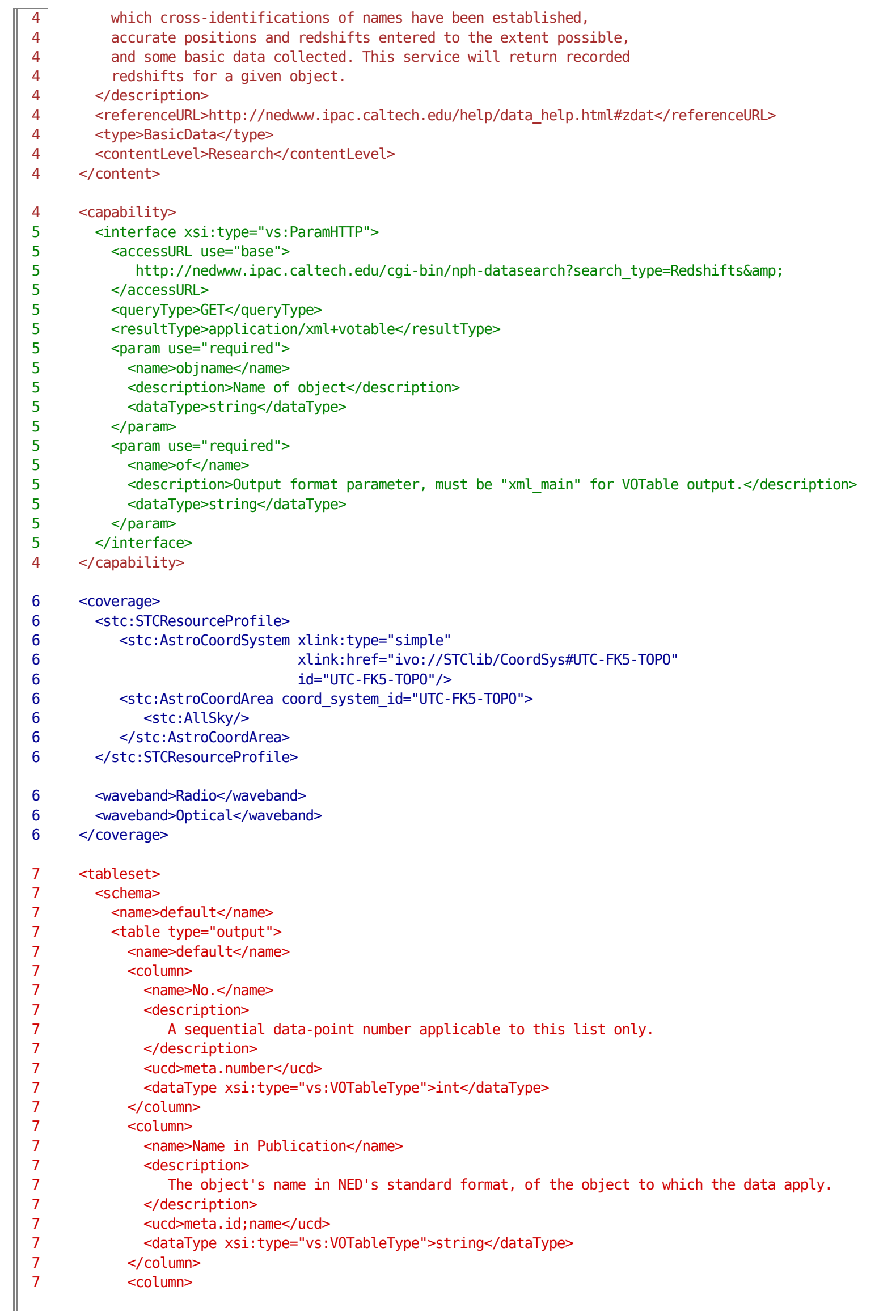




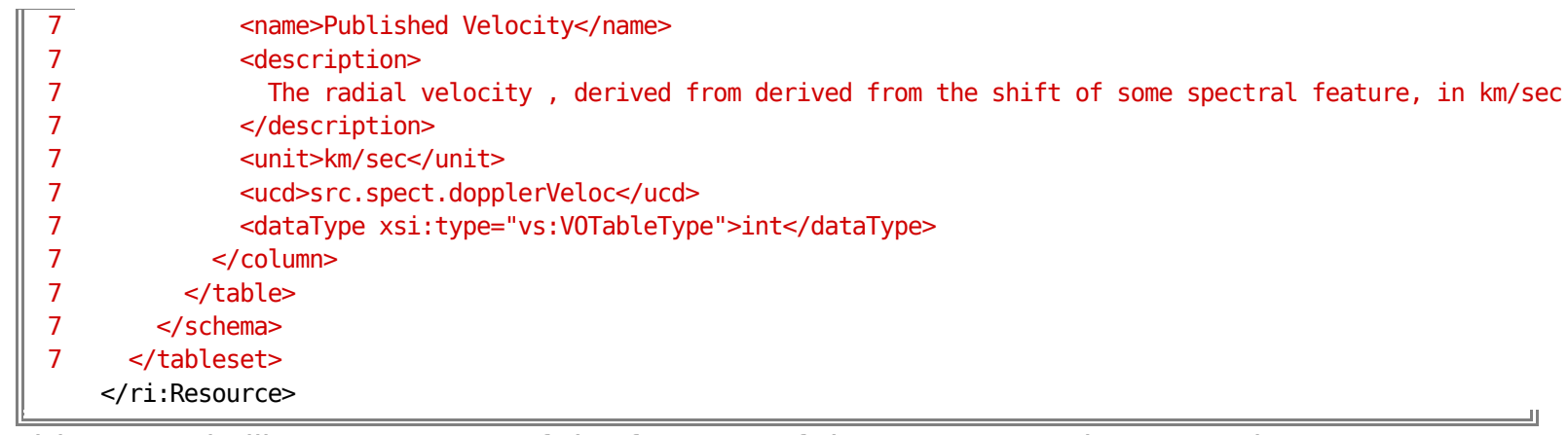

This example illustrates some of the features of the VODataService extension:

1. the extra namespaces associated with VODataService metadata; if STC coverage information [STC] is not included, then only the VODataService namespaced is needed.

2. the specific type of resource indicated by the value of the xsi: type attribute; in this case vs: CatalogService indicates that this is describing a service that accesses tabular data.

3. the location of the VOResource-related schema documents used by this description,

4. the core VOResource metadata,

5. an interface described by the VODataService interface type, vs:ParamHTTP; this type can indicate input arguments it supports.

6. a description of the coverage, including an STC description plus waveband keywords.

7. a description of the table that is returned by the service.

\subsection{The Schema Namespace and Location}

The namespace associated with VODataService extensions is "http://www.ivoa.net /xmI/VODataService/v1.1". Just like the namespace URI for the VOResource schema, the VODataService namespace URI can be interpreted as a URL. Resolving it will return the XML Schema document (given in Appendix A) that defines the VODataService schema.

Authors of VOResource instance documents may choose to provide a location for the VOResource XML Schema document and its extensions using the xsi:schemaLocation attribute. While the choice of the location value is the choice of the author, this specification recommends using the VODataService namespace URI as its location URL (as illustrated in the example above), as in,

xsi:schemaLocation="http://www. ivoa. net/xml/VODataService/v1.1 http://www. ivoa. net/xml/VODataService/v1.1"

\section{Note:}

The IVOA Registry Interface standard [RI] actually requires that the VOResource records it shares with other registries provide location URLS via xsi:schemaLocation for the VOResource schema and all legal extension schemas that are used in the records. This rule would apply to the vODataService schema.

The prefix, vs, is used by convention as the prefix defined for the VODataService schema; however, instance documents may use any prefix. In applications where common use of prefixes is recommended (such as with the Registry Interface specification [RI]), use of the vs prefix is recommended. Note also that in this document, the vr prefix is used to label, as shorthand, a type or element name that is defined in the VOResource schema, as 
in vr: Resource.

Note:

One reason one may not be able to use vs to represent the

VODataService schema, version 1.1 , is because it is already in defined to represent VODataService v1.0 and cannot be overridden. At this writing, there are no IVOA applications in which this is the case. Consult Appendix B for more details on compatibility issues.

As recommend by the VOResource standard [VOR], the VODataService schema sets elementFormDefault="unqualified". This means that it is not necessary to qualify element names defined in this schema with a namespace prefix (as there are no global elements defined). The only place it is usually needed is as a qualifier to a VODataService type name given as the value of an xsi:type attribute.

\subsection{Summary of Metadata Concepts}

The VODataService extension defines four new types of resources. Two inherit directly from vr:Resource:

vs: DataCollection

This resource declares the existence of a collection of data, what it represents, and how to get it. The access to the data may be limited to a human-readable web page (given by content/referenceURL); however, if the contents of the collection are available statically via a URL (e.g. an FTP URL to a directory containing all the files), that URL can be provided. It can also provide pointers to other IVOA registered services that can be used to access the data.

vs: StandardSTC

This resource type declares one or more coordinate systems described using STC [STC] such that each can be assigned a globally unique identifier (based on the IVOA identifier for the resource record itself). This identifier can then be referenced in any other STC description in lieu of a fully described coordinate system. Coordinate systems described in this way become reusable standards once they are registered in an IVOA registry.

The other two resource types represent specialized services:

vs:DataService

Inheriting from vr:Service, this type is for services that access astronomical data. It adds the ability to describe the data's coverage of the sky, frequency, and time.

vs: CatalogService

Inheriting from vs:DataService, this type specifically refers to a service that accesses tabular data. In addition to the coverage information, this type adds the ability to describe the tables and their columns. This is intended for describing services that support the "simple" IVOA data access layer protocols such as Simple Image Access [SIA] and Simple Cone Search [SCS].

In general, coverage refers to the extent that data samples the measurement range of the 
sky (space), frequency, and time. The coverage metadata (encoded via the vs: coverage type) has two parts. The first part allows a full STC profile description (via the imported STC element, <stc:STCResourceProfile>). The second part captures key coverage metadata defined in the IVOA Resource Metadata standard [RM]. The RM-derived coverage elements can be considered summarizing metadata for many of the details that may appear within the STC description, and enables simpler searching of high-level coverage information.

The detailed STC profile contained within the <stc: STCResourceProfiles element is capable of describing coverage not only in space, time, and frequency but also velocity and redshift. The profile contains up to three types of component descriptions ([STC], section 4.1): coordinate systems, coordinate values, and coordinate areas or ranges. The first component describes the coordinate systems to which coordinate values, areas, and regions are referenced. While any arbitrary system can be described in this first part, it is expected that most VODataService instances will provide a simple pointer to a predefined system in a registered vs: Standardstc record (using the mechanism summarized in section 3.1.2 below). The coordinate values part will usually be used to describe the coordinate resolution, errors, or typical sizes. The coordinate areas part describes actual regions or ranges covered by the resource in any of the given coordinate systems.

Table descriptions appear within a single <tableset> element. This element can in turn can contain one or more sschemas element in which each schema represents a set of logically related tables. It is not required that that the schema grouping match the underlying database's catalogs or schemas (as defined in [SQLGuide]), though it may. In some cases, such as when describing the table that is returned from an SIA service, the terms catalog and schema may have little relevance; in this case, the table can be considered part of a sole "default" schema.

For each table in a schema, one can describe each of the columns, providing such information as its name, type, UCD [ $\underline{U C D}$ ], units, and a textual description. Providing this information makes it possible to select a resource based on the kind data contained in its tables.

Finally, the VODataService defines specialized interface type (inheriting from vr: Interface) called vs:ParamHTTP. This type is used to describe the commonly used interface that is invoked over HTTP as either a GET or a POST [HTTP] in which the arguments are encoded as name=value pairs. In addition to the access URL, it can include not only the mime-type of the returned response, it can also enumerate the input arguments that are supported by the service implementation. Much like table columns, one can indicate for each argument the name, the UCD, the data type, the units, whether it is required, and a textual description of the argument. Note that this does not capture any interdependencies between arguments. For example, it cannot indicate if one argument only makes sense in the presence of another argument.

\section{The VODataService Metadata}

This section enumerates the types and elements defined in the VODataService extension schema and describes their meaning. Where a term matches a term in the $\underline{R M}$, its meaning is given in terms of the RM definition.

\subsection{Resource Type Extensions}

\subsubsection{DataCollection}


A data collection, which is describable with the vs: Datacollection resource type, is a logical group of data comprising one or more accessible datasets. A collection can contain any combination of images, spectra, catalogs, time-series, or other data. (In contrast, we talk about a dataset as being a set of digitally-encoded data that is normally accessible as a single unit--e.g., a file.)

The vs:Datacollection type adds seven additional metadata elements beyond the core VOResource metadata [VOR].

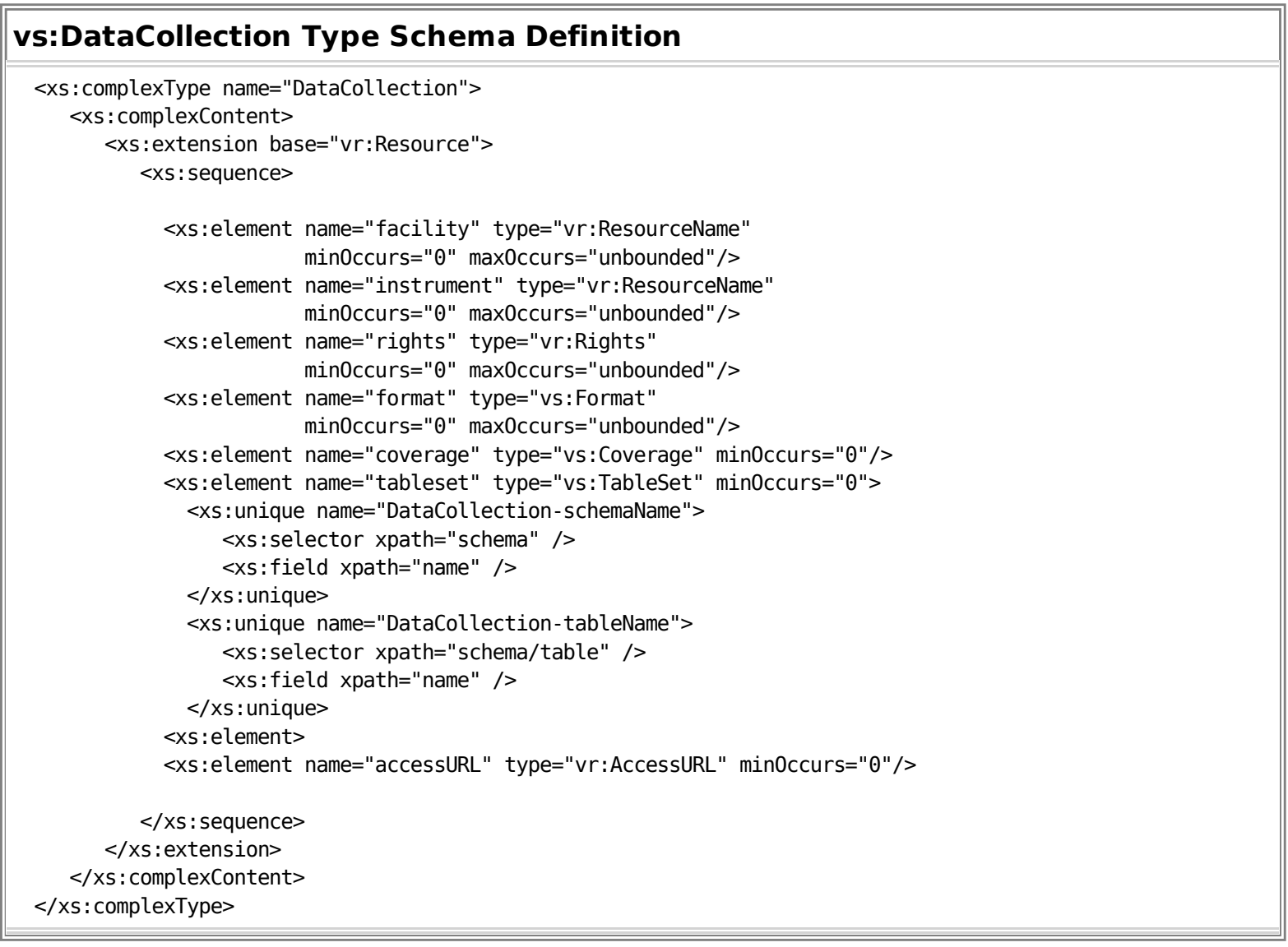

The definition of <tableset> element places forces certain names within its description to be unique; these constraints are explained further in 3.3.1.

All of the child elements except <tableset> derive from RM terms. Four of the elements--<facility>, <instrument>, <rights>, and <accessURL>--are reuses of elements defined in the core VOResource schema, sharing the same syntax and similar semantics. In particular, the meanings of <facility> and <instrument> in the context of vs:Datacollection are different from that in vr:Organisation only in that in the former type, they refer to the origin of the data.

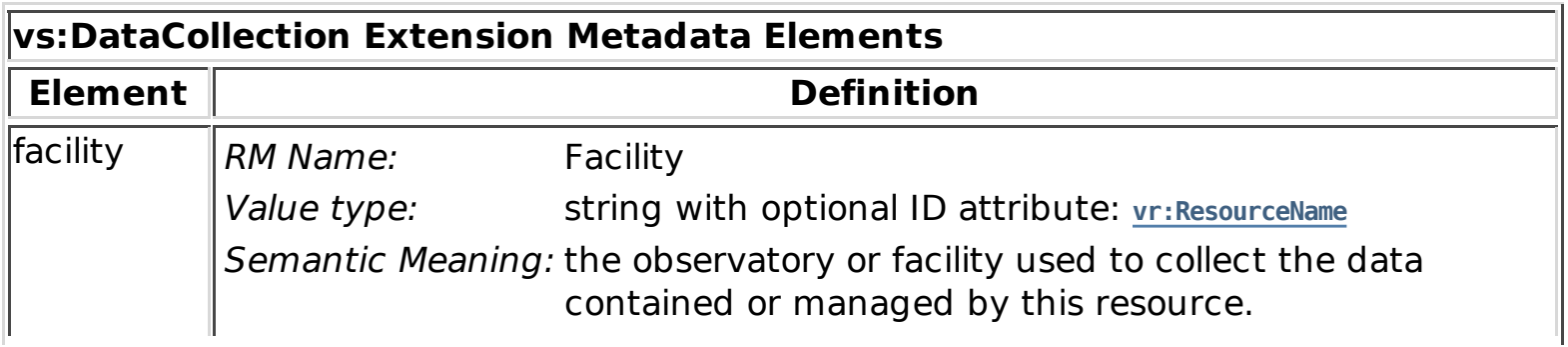




\begin{tabular}{|c|c|c|}
\hline \multirow[t]{2}{*}{ Element } & \multicolumn{2}{|r|}{ Definition } \\
\hline & Occurrences: & optional; multiple occurrences allowed \\
\hline instrument & $\begin{array}{l}\text { RM Name: } \\
\text { Value type: } \\
\text { Semantic Meaning: } \mathrm{t} \\
\text { Occurrences: }\end{array}$ & $\begin{array}{l}\text { Instrument } \\
\text { string with optional ID attribute: vr:ResourceName } \\
\text { the instrument used to collect the data contained or } \\
\text { managed by this resource. } \\
\text { optional; multiple occurrences allowed }\end{array}$ \\
\hline rights & $\begin{array}{l}\text { RM Name: } \\
\text { Value type: } \\
\text { Semantic Meaning: I } \\
\text { Occurrences: } \\
\text { Allowed Values: }\end{array}$ & $\begin{array}{l}\text { Rights } \\
\text { string, controlled vocabulary: xs: token } \\
\text { Information about rights held in and over the resource. } \\
\text { optional; multiple occurrences allowed } \\
\text { public unrestricted, public access is allowed without } \\
\text { authentication. } \\
\text { secure authenticated, public access is allowed. } \\
\text { proprietary only proprietary access is allowed with } \\
\quad \text { authentication. }\end{array}$ \\
\hline format & $\begin{array}{l}\text { RM Name: } \\
\text { Value type: } \\
\text { Semantic Meaning: } \\
\text { Occurrences: } \\
\text { Comments: }\end{array}$ & $\begin{array}{l}\text { Format } \\
\text { string with optional isMIMEType attribute, vs:Format } \\
\text { The physical or digital manifestation of the information } \\
\text { supported by a resource. } \\
\text { optional; multiple occurrences allowed } \\
\text { MIME types should be used for network-retrievable, } \\
\text { digital data, and the isMIMEType attribute should be set to } \\
\text { explicitly to "true". Non-MIME type values are used for } \\
\text { media that cannot be retrieved over the network--e.g. } \\
\text { CDROM, poster, slides, video cassette, etc. }\end{array}$ \\
\hline coverage & $\begin{array}{l}\text { RM Name: } \\
\text { Value type: } \\
\text { Semantic Meaning: } \\
\text { Occurrences: }\end{array}$ & $\begin{array}{l}\text { Coverage } \\
\text { composite; vs: Coverage } \\
\text { Extent of the content of the resource over space, time, } \\
\text { and frequency. } \\
\text { optional }\end{array}$ \\
\hline tableset & $\begin{array}{l}\text { Value type: } \\
\text { Semantic Meaning: } \\
\text { Occurrences: }\end{array}$ & $\begin{array}{l}\text { composite; vs: Tableset } \\
\text { A description of tables that are part of this collection. } \\
\text { optional. }\end{array}$ \\
\hline accessURL & $\begin{array}{l}\text { RM Name: } \\
\text { Value type: } \\
\text { Semantic Meaning: } \\
\text { Occurrences: }\end{array}$ & $\begin{array}{l}\text { Service. AccessURL } \\
\text { URL with optional use attribute: vr: AccessURL } \\
\text { The URL can be used to download the data contained in } \\
\text { this data collection. } \\
\text { required; multiple occurrences allowed. }\end{array}$ \\
\hline
\end{tabular}

The vs: Format type is used for providing a value to the <format> element: 


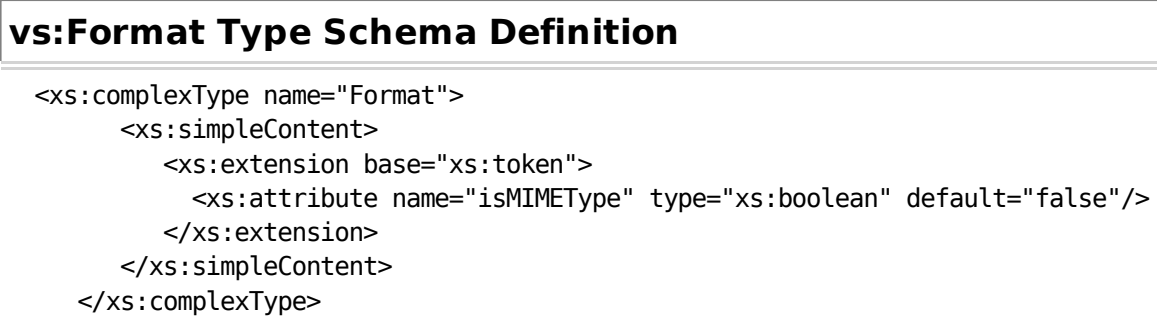

The isMIMEType attribute provides a flag to indicate if the value represents an actual MIME-type: if it is, this attribute should be explicitly set to "true".

See section 3.3 for a specification of the vs: Tableset type for describing tables.

\subsubsection{StandardSTC}

The vs:StandardSTc resource type is used to register standard coordinate systems, positions, or regions using the Space-Time Coordinate (STC, [STC]) standard schema so that they can by uniquely referenced by name by other resource descriptions or applications. This resource type extends the core metadata with a single element, <stcDefinitions>, which contains the STC definitions.

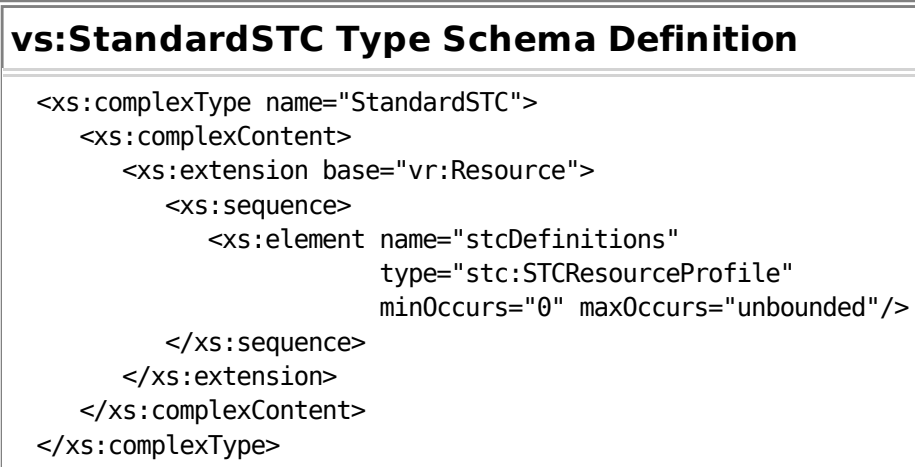

The curation metadata that is part of the core VODataService should generally refer to the publishing organization and persons that are responsible for defining the systems, updating the definitions as needed, and responding to user questions about the definitions. The content metadata, in particular the textual contents of the <description> element, should describe the purpose of the definition and where references to the defined systems, positions, or regions may be used.

\begin{tabular}{|l|lr|}
\hline \multicolumn{2}{|l|}{ vs:StandardSTC Extension Metadata Elements } \\
\hline Element & \multicolumn{1}{c|}{ Definition } \\
\hline stcDefintions & $\begin{array}{l}\text { Value type: } \\
\text { Semantic Meaning: }\end{array}$ & $\begin{array}{r}\text { composite; stc:stcDescriptiontype } \\
\text { are available for referencing. } \\
\end{array}$ \\
& Occurrences: & required; multiple occurrences allowed \\
\hline
\end{tabular}

The content of the <stcDefinitions s element is controlled by the STC schema. Because that schema uses the elementFormDefault="true" and most of the STC elements are defined to be global [schema], <stcDefinitions> child elements must be qualified as being in the STC namespace (http://www.ivoa.net/xml/STC/stc-v1.30.xsd), by either setting the default 
namespace (via the xmlns attribute) or via explicit qualification via a prefix (see example).

\subsubsection{DataService}

The vs:DataService resource type is for describing a service that provides access to astronomical data. This service adds to the core VOResource service metadata the ability to associate an observing facility and/or instrument with the data as well as describe the coordinate coverage of data via its child <coverages element. Note that while these elements are all optional, a resource of this type still implies access to astronomical data.

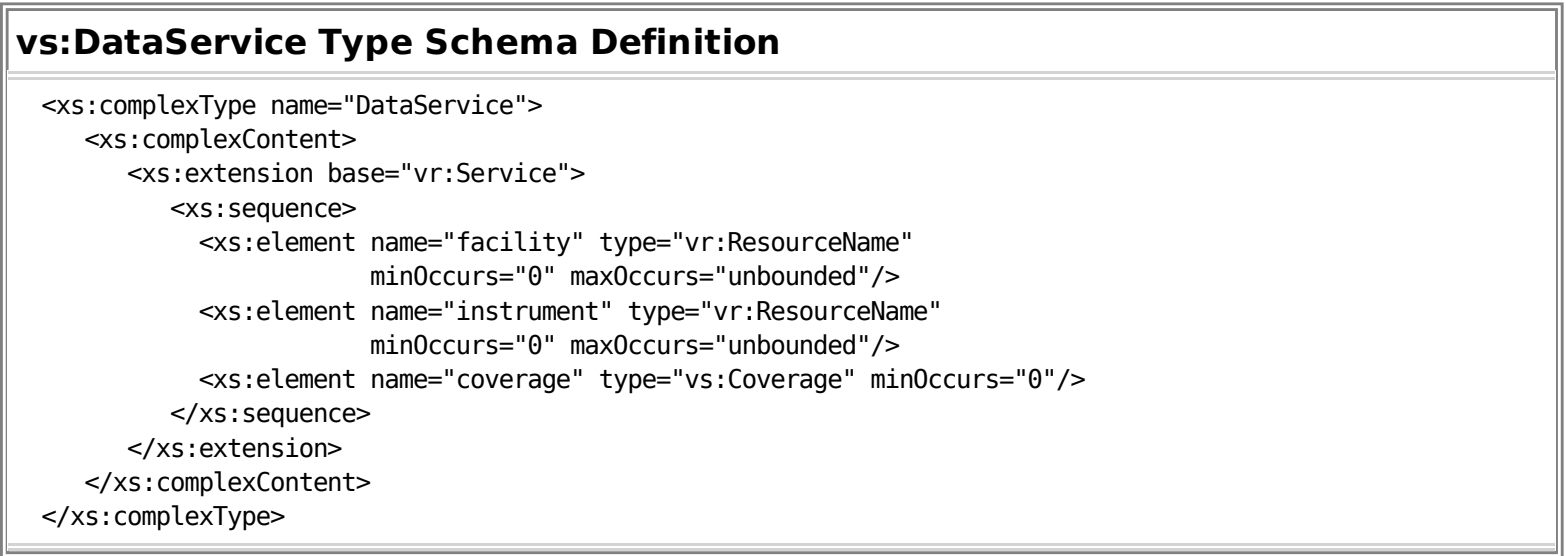

The use and meaning of the <facility> and <instrument> elements are the same as for vs: Datacollection.

\begin{tabular}{|c|c|c|}
\hline \multicolumn{3}{|c|}{ vs:DataService Extension Metadata Elements } \\
\hline Element & & Definition \\
\hline facility & $\begin{array}{l}\text { RM Name: } \\
\text { Value type: } \\
\text { Semantic Meaning: } \\
\text { Occurrences: }\end{array}$ & $\begin{array}{l}\text { Facility } \\
\text { string with optional ID attribute: vr:ResourceName } \\
\text { the observatory or facility used to collect the data } \\
\text { contained or managed by this resource. } \\
\text { optional; multiple occurrences allowed }\end{array}$ \\
\hline instrument & $\begin{array}{l}\text { RM Name: } \\
\text { Value type: } \\
\text { Semantic Meaning: } \\
\text { Occurrences: }\end{array}$ & $\begin{array}{l}\text { Instrument } \\
\text { string with optional ID attribute: vr:ResourceName } \\
\text { the instrument used to collect the data contained or } \\
\text { managed by this resource. } \\
\text { optional; multiple occurrences allowed }\end{array}$ \\
\hline coverage & $\begin{array}{l}\text { RM Name: } \\
\text { Value type: } \\
\text { Semantic Meaning: } \\
\text { Occurrences: }\end{array}$ & $\begin{array}{l}\text { Coverage } \\
\text { composite; vs: Coverage } \\
\text { Extent of the content of the resource over space, time, } \\
\text { and frequency. } \\
\text { optional }\end{array}$ \\
\hline
\end{tabular}

The contents of the <coverages element are detailed in section 3.2.

\subsubsection{CatalogService}


The vs: Catalogservice resource type is for describing a service that interacts with astronomical data through one or more specified tables. Because it extends the vs:DataService type, a catalog service can have a coverage description as well. The tabular data may optionally be described via a <tableset> element.

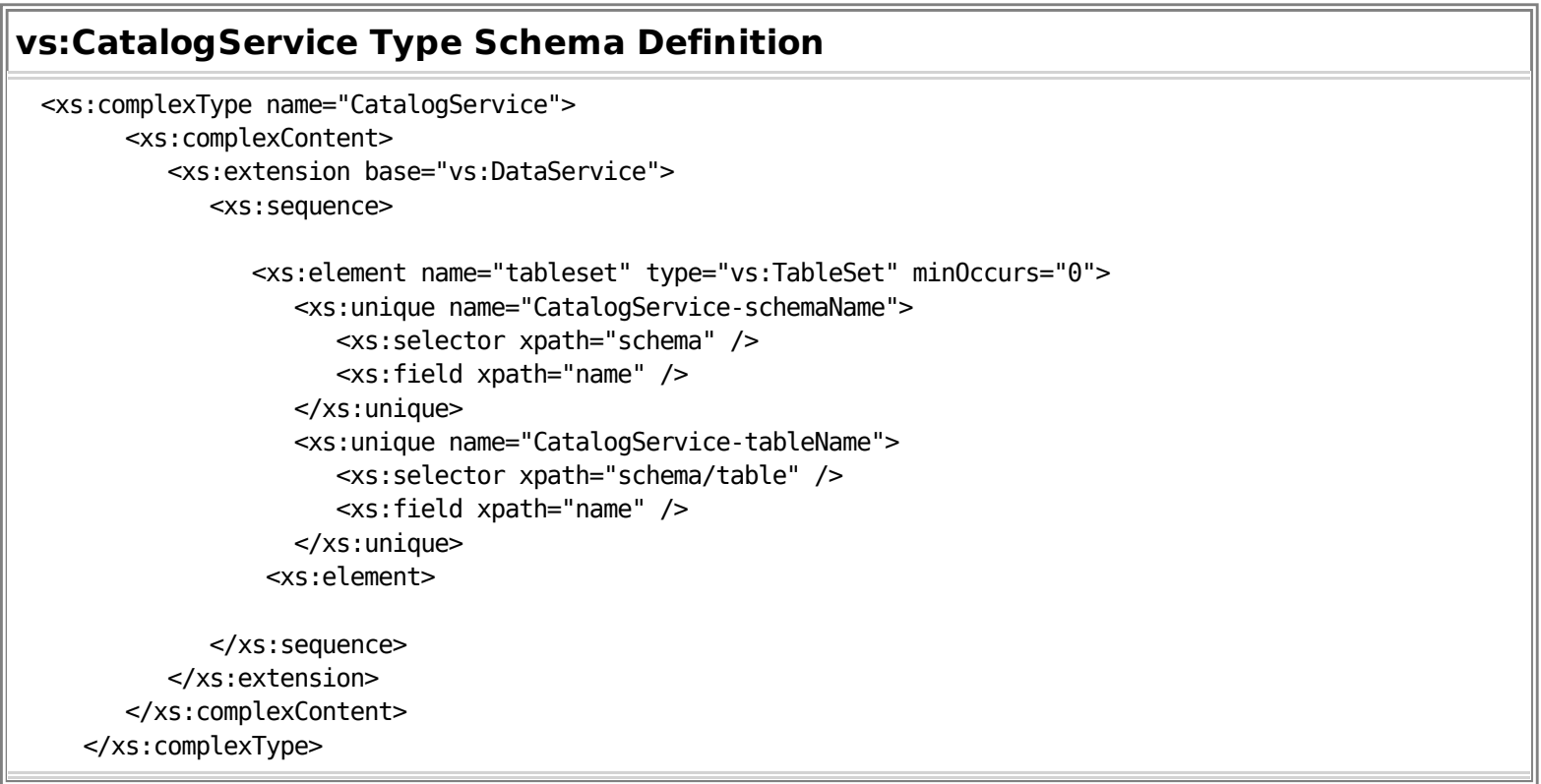

The definition of <tableset> element forces certain names within its description to be unique; these constraints are explained further in 3.3.1.

\begin{tabular}{|l|l|}
\hline vs: CatalogService Extension Metadata Elements \\
\hline Element & \multicolumn{1}{c|}{ Definition } \\
\hline tableset & $\begin{array}{ll}\text { Value type: } & \text { composite; vs: Tableset } \\
\text { Semantic Meaning: A description of the tables that are accessible through this } \\
\text { service. } \\
\text { optional }\end{array}$ \\
\hline
\end{tabular}

\subsection{Coverage}

The vs: Coverage type describes how the data samples the sky, frequency, and time.

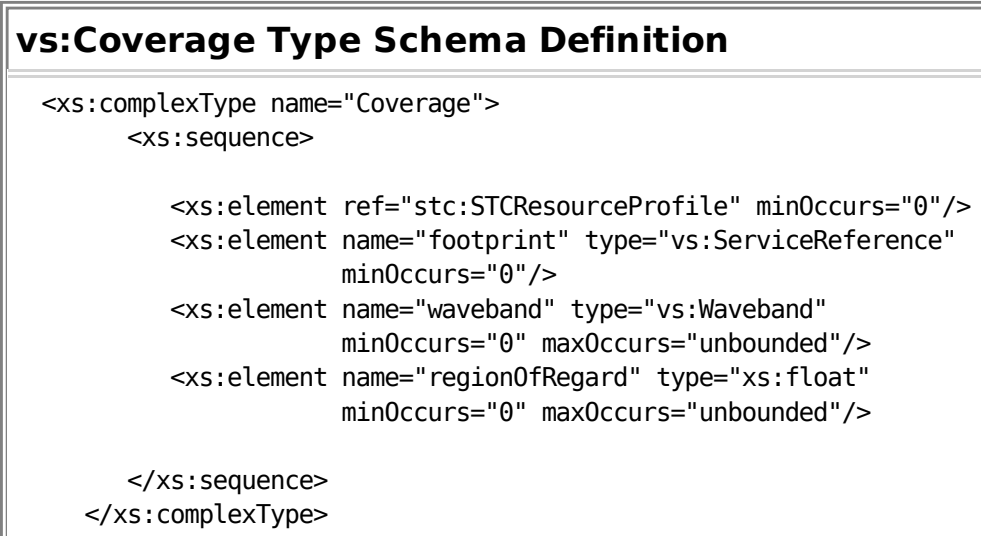


A detailed, systematic description of coverage is provided via the child <stc:STCResourceProfile> element, taken from the STC schema, version 1.3, with the namespace, http://wmw.ivoa.net/xml/STc/stc-v1.30.xsd (hereafter referred using the stc: prefix). This element is defined in the STC schema as a global element; furthermore, the STC schema sets its global elementFormDefault="qualified". Consequently, the <stc: STCResourceProfile> element and all its child elements must be qualified as part of the STC namespace as required by XML Schema [schema]. In applications where common use of XML prefixes is required or encouraged (e.g. the IVOA Registry Interfaces [ $\underline{R I}]$ ), use of the stc: prefix to represent the STC namespace is encouraged.

\section{Note:}

The STC scheme provides rich mark-up for expressing the details of the coverage. In particular, the mark-up is quite flexible in the units that can be used. For example, spectral coverage can be given in terms of frequency, wavelength, or energy. While it is recommended that the overall description given in the <stc: STCResourceProfile> be fairly general and approximate, leveraging the richness for a detailed description is allowed.

The remaining elements provide some summary information about the coverage.

\begin{tabular}{|c|c|c|}
\hline \multicolumn{3}{|c|}{ vs:Coverage Metadata Elements } \\
\hline Element & & Definition \\
\hline STCResourceProfile & $\begin{array}{l}\text { Value type: } \\
\text { Semantic Meaning: } \\
\text { Occurrences: } \\
\text { Comments: }\end{array}$ & $\begin{array}{l}\text { composite: an stc:STCResourceProfile element from } \\
\text { the STC schema. } \\
\text { The STC description of the location of the } \\
\text { resource's data (or behavior on data) on the sky, } \\
\text { in time, and in frequency space, including } \\
\text { resolution. } \\
\text { optional } \\
\text { In general, this description should be } \\
\text { approximate; a more precise description can be } \\
\text { provided by the service referred to by the } \\
\text { <footprint> element. }\end{array}$ \\
\hline footprint & $\begin{array}{ll}\text { Value type: } & \\
\text { Semantic Meaning: } & \\
\text { Occurrences: } & \\
\text { Comments: } & \end{array}$ & $\begin{array}{l}\text { a URL with an optional IVOA identifier attribute: } \\
\text { a reference to a footprint service for retrieving } \\
\text { precise and up-to-date description of coverage. } \\
\text { optional } \\
\text { the ivo-id attribute refers to a Service record } \\
\text { having a footprint service capability. That is, the } \\
\text { record will have a capability element describing } \\
\text { the footprint service (see "Note on Footprint } \\
\text { Service" below for further discussion). The } \\
\text { resource referred to may be the current one. }\end{array}$ \\
\hline waveband & $\begin{array}{l}\text { RM Name: } \\
\text { Value type: }\end{array}$ & $\begin{array}{l}\text { Coverage.Spectral } \\
\text { string with controlled vocabulary: vs:Waveband }\end{array}$ \\
\hline
\end{tabular}




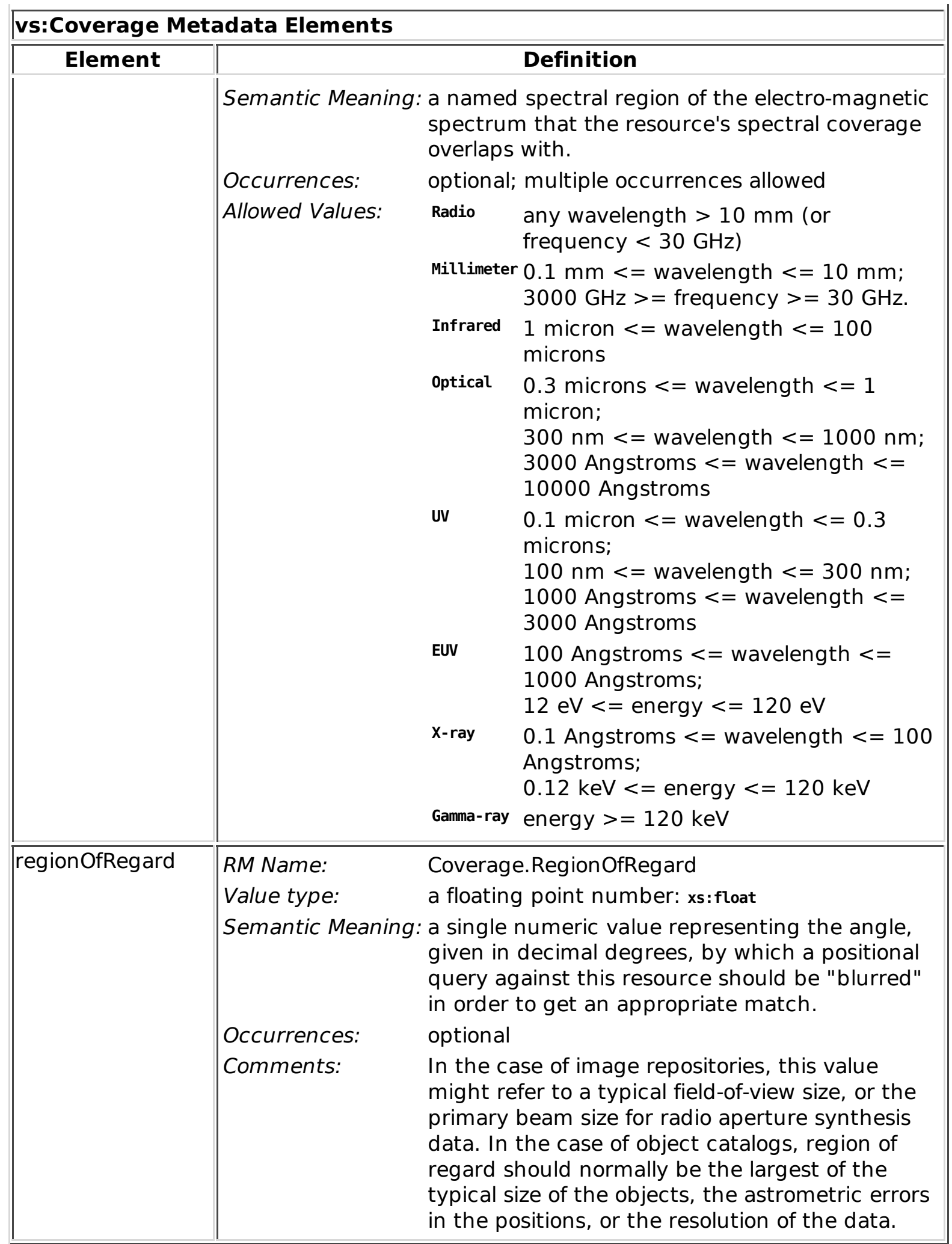

Note on Footprint Service:

The <footprint> element has been defined in anticipation of a future standard IVOA footprint service protocol that can be used to respond to 
detailed spatial overlap queries. Consequently, in the future, applications may be able to assume the protocol that footprint service URL supports. When an application is unable to make any assumptions, the IVOA Identifier given by the attribute should be resolved and the returned resource description should be searched for a recognized footprint service capability.

\subsection{Tabular Data}

The vs: Tableset type can be used to describe a set of tables that are part of a single resource and can be consider functionally all located at a single site.

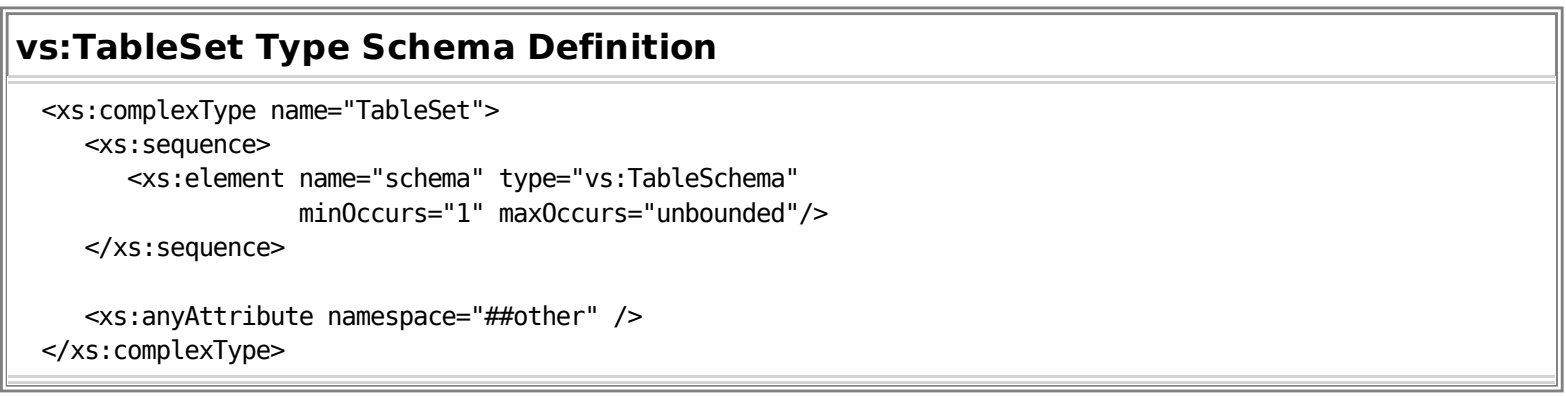

\begin{tabular}{|c|c|c|}
\hline \multicolumn{3}{|c|}{ vs:TableSet Metadata Elements } \\
\hline Element & & Definition \\
\hline schema & $\begin{array}{l}\text { Value type: } \\
\text { Semantic Meaning: } \\
\text { Occurrences: } \\
\text { Comments: }\end{array}$ & $\begin{array}{l}\text { composite; vs: Tableschema } \\
\text { A named description of a set of logically related tables. } \\
\text { required; multiple occurrences are allowed. } \\
\text { See section 3.3.1 regarding unique names for schemas. }\end{array}$ \\
\hline
\end{tabular}

The vs: Tableschema type collects tables together that are logically related. For example, a single resource may provide access several major astronomical catalogs (e.g. SDSS, 2 MASS, and FIRST) from one site, enabling high-performance cross-correlations between them. Each catalog can be described in a separate <schemas element, using the elements from the vs: Tableschema type.

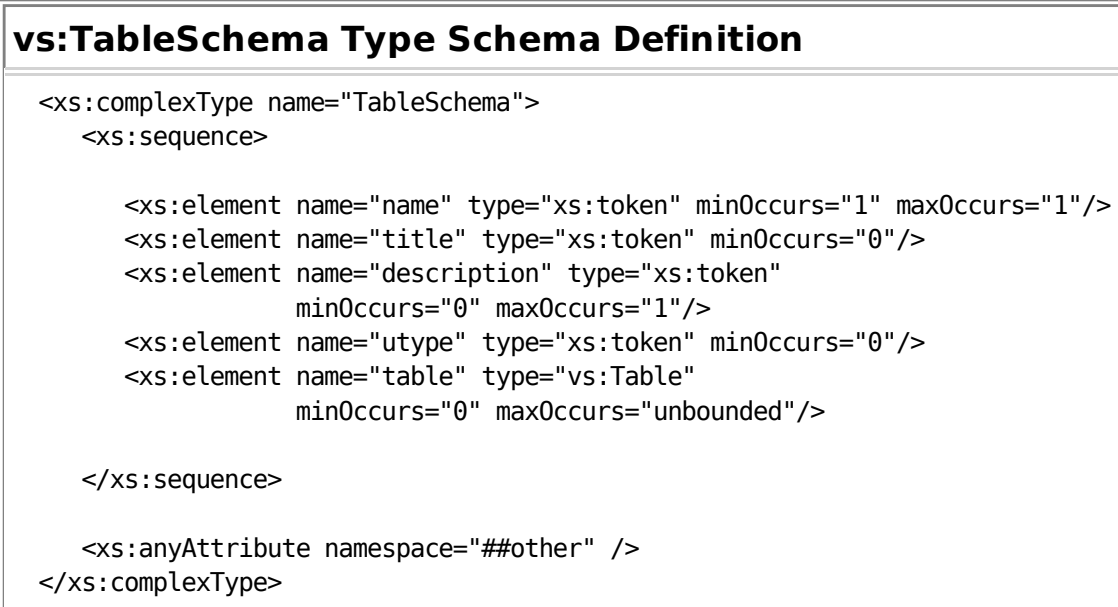




\begin{tabular}{|c|c|c|}
\hline \multicolumn{3}{|c|}{ vs:TableSchema Metadata Elements } \\
\hline Element & & Definition \\
\hline name & $\begin{array}{l}\text { Value type: } \\
\text { Semantic Meaning: } \\
\text { Occurrences: } \\
\text { Comments: }\end{array}$ & $\begin{array}{l}\text { string: xs: token } \\
\text { A name for the set of tables. } \\
\text { required } \\
\text { If there is no appropriate logical name associated with } \\
\text { this set, the name should be explicitly set to "default". } \\
\text { See section 3.3.1 regarding the uniqueness of this } \\
\text { name. }\end{array}$ \\
\hline title & $\begin{array}{l}\text { Value type: } \\
\text { Semantic Meaning: } \\
\text { Occurrences: } \\
\text { Comments: }\end{array}$ & $\begin{array}{l}\text { string: xs: token } \\
\text { a descriptive, human-interpretable name for the table } \\
\text { set. } \\
\text { optional } \\
\text { This is used for display purposes and is useful when } \\
\text { there are multiple schemas in the context (e.g. within a } \\
\text { tableset; otherwise, the resource title could be used } \\
\text { instead). Note, however, that there is no requirement } \\
\text { regarding uniqueness. If a title is not provided, the } \\
\text { schema name can be used for display purposes. }\end{array}$ \\
\hline description & $\begin{array}{l}\text { Value type: } \\
\text { Semantic Meaning: } \\
\text { Occurrences: }\end{array}$ & $\begin{array}{l}\text { string: xs: token } \\
\text { A free text description of the tableset that should } \\
\text { explain in general how all of the tables are related. } \\
\text { optional }\end{array}$ \\
\hline utype & $\begin{array}{l}\text { Value type: } \\
\text { Semantic Meaning: } \\
\text { Occurrences: } \\
\text { Comments: }\end{array}$ & $\begin{array}{l}\text { String: xs: token } \\
\text { an identifier for a concept in a data model that the data } \\
\text { in this schema as a whole represent. } \\
\text { optional } \\
\text { The format defined in the VOTable standard, section } 4.1 \\
\text { [VOTable] is strongly recommended; see "Note on } \\
\text { UType Format below. }\end{array}$ \\
\hline table & $\begin{array}{l}\text { Value type: } \\
\text { Semantic Meaning: } \\
\text { Occurrences: } \\
\text { Comments: }\end{array}$ & $\begin{array}{l}\text { composite: vs: Table } \\
\text { A marked description of one of the tables that makes up } \\
\text { the set. } \\
\text { optional; multiple occurrences are allowed. } \\
\text { See section 3.3.1 regarding unique names for schemas. }\end{array}$ \\
\hline
\end{tabular}

Note on UType Format:

As of this writing, an IVOA standard for the format of utypes is still under development. As a result, the most definitive documentation of the format is in section 4.1 of the VOTable specification [VOTable], which is expected to be a more general form to be spelled out in the eventual utype standard. Use of that latter standard is recommended once it becomes available.

Each table in a schema is described in detail using the vs: Table type. 


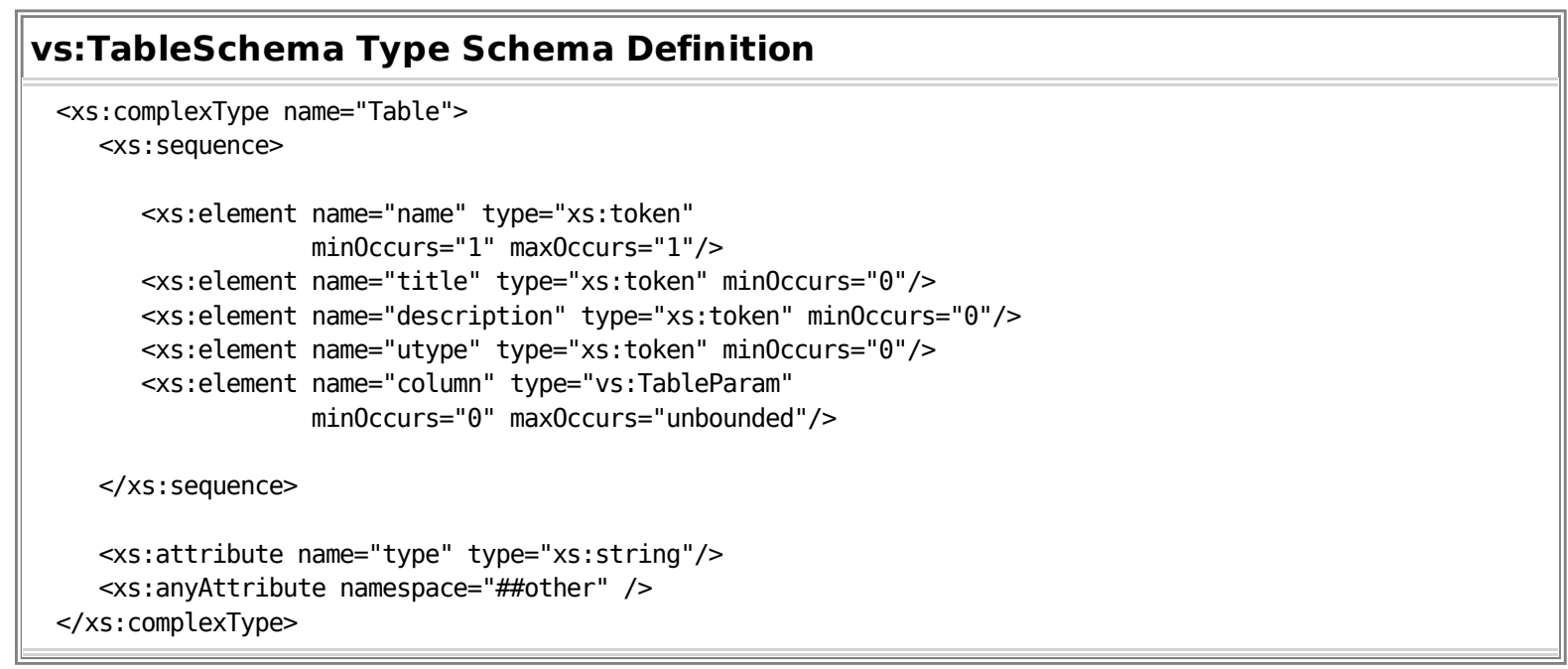

\begin{tabular}{|c|c|c|}
\hline \multicolumn{3}{|c|}{ vs:Table Metadata Elements } \\
\hline Element & & Definition \\
\hline name & $\begin{array}{l}\text { Value type: } \\
\text { Semantic Meaning: } \\
\text { Occurrences: } \\
\text { Comments: }\end{array}$ & $\begin{array}{l}\text { string: xs: token } \\
\text { A fully qualified name for the table. This name should } \\
\text { include all catalog or schema prefixes needed to } \\
\text { sufficiently uniquely distinguish it in a query to the } \\
\text { table. } \\
\text { required } \\
\text { In general, the format of the qualified name may } \\
\text { depend on the on the context; however, when the table } \\
\text { is intended to be queryable via ADQL [ADQL], then the } \\
\text { catalog and schema qualifiers are delimited from the } \\
\text { table name with dots (.). } \\
\text { If this table is part of the schema named "default", the } \\
\text { schema name does not need to appear in this table } \\
\text { name, unless it is required by an associated access } \\
\text { service. } \\
\text { If there is no appropriate logical name associated with } \\
\text { this table, the name should be explicitly set to "default". } \\
\text { See section 3.3.1 regarding the uniqueness of this } \\
\text { name. }\end{array}$ \\
\hline title & $\begin{array}{l}\text { Value type: } \\
\text { Semantic Meaning: } \\
\text { Occurrences: } \\
\text { Comments: }\end{array}$ & $\begin{array}{l}\text { string: xs: token } \\
\text { a descriptive, human-interpretable name for the table } \\
\text { set. } \\
\text { optional } \\
\text { This is used for display purposes. There is no } \\
\text { requirement regarding uniqueness. If a title is not } \\
\text { provided, the table name can be used for display } \\
\text { purposes. }\end{array}$ \\
\hline description & $\begin{array}{l}\text { Value type: } \\
\text { Semantic Meaning }\end{array}$ & $\begin{array}{l}\text { string: xs: token } \\
\text { A free-text description of the table's contents. }\end{array}$ \\
\hline
\end{tabular}




\begin{tabular}{|c|c|c|}
\hline \multicolumn{3}{|c|}{ vs:Table Metadata Elements } \\
\hline Element & & Definition \\
\hline & Occurrences: & optional \\
\hline utype & $\begin{array}{l}\text { Value type: } \\
\text { Semantic Meaning: } \\
\text { Occurrences: } \\
\text { Comments: }\end{array}$ & $\begin{array}{l}\text { string: xs: token } \\
\text { an identifier for a concept in a data model that the data } \\
\text { in this table as a whole represent. } \\
\text { optional } \\
\text { The format defined in the VOTable standard, section } 4.1 \\
\text { [VOTable] is strongly recommended; see "Note on } \\
\text { UType Format above. }\end{array}$ \\
\hline column & $\begin{array}{l}\text { Value type: } \\
\text { Semantic Meaning: } \\
\text { Occurrences: } \\
\text { Comments: }\end{array}$ & $\begin{array}{l}\text { composite: vs:TableParam } \\
\text { A marked description of one of the table's columns. } \\
\text { optional; multiple occurrences are allowed. } \\
\text { See section } 3.5 \text { for the description of this element's } \\
\text { contents. }\end{array}$ \\
\hline foreignKey & $\begin{array}{l}\text { Value type: } \\
\text { Semantic Meaning: } \\
\text { Occurrences: } \\
\text { Comments: }\end{array}$ & $\begin{array}{l}\text { composite: vs: Foreignkey } \\
\text { A description of a foreign keys, one or more columns } \\
\text { from the current table that can be used to join with } \\
\text { another table. } \\
\text { optional; multiple occurrences are allowed. } \\
\text { See section 3.5.2 for the description of this element's } \\
\text { contents. }\end{array}$ \\
\hline
\end{tabular}

Each column in a table can be described using the vs: TableParam type which is described in section 3.5. The foreign keys in the table that can be used to join it with another table can be described with the vs: ForeignKey type (section 3.3.2). A foreign key description should only refer to tables described within the current table set.

The vs: Table also provides an attribute for indicating the role a table plays in the schema:

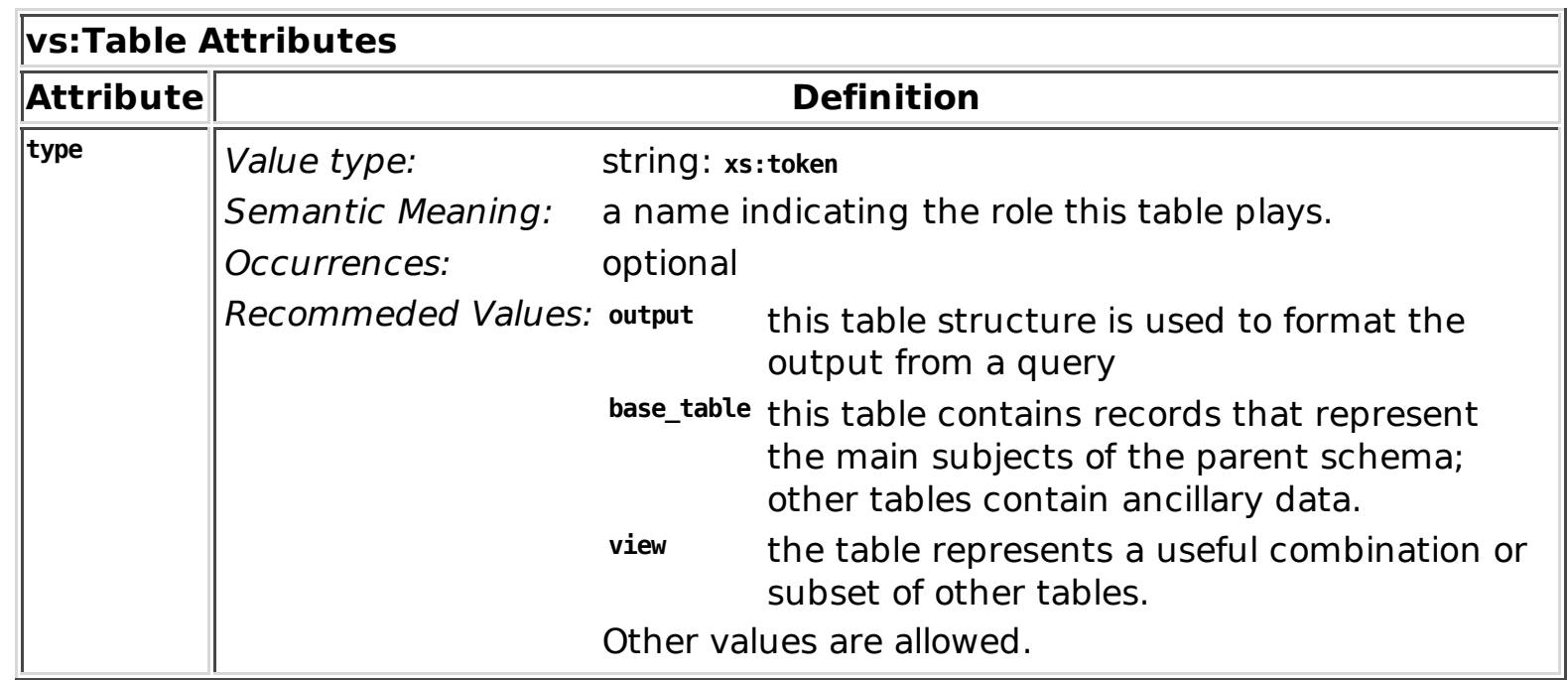

\subsubsection{Unique Names for Tables}


The definitions of the <tableset> elements used in the vs:Datacollection and vs: CatalogService types constrain certain names to be unique. In particular, all schema names within a <tableset> element must be unique, and all table names within a <tableset> element must be unique. (A schema and table may share a common name, such as "default".) These constraints makes it possible to uniquely locate the description of a schema or table within a VOResource description.

\section{Example}

The uniqueness constraints for names within table sets guarantee that when the following XPath queries are applied to a <tableset> element, zero or one node only will be returned:

schema [@name="default"]

schema/table [@name="default"]

Name uniqueness is only required when the table set description is part of a VOResource description. The name uniqueness rules should also be applied to other uses of the vs: Tableset element outside of a VOResource description.

\subsubsection{Foreign Keys}

The vs: Foreignkey type allows one to describe foreign keys in a table that allow it to be joined effectively with another table. A foreign key is a set of columns that map to a corresponding set of columns in another table.

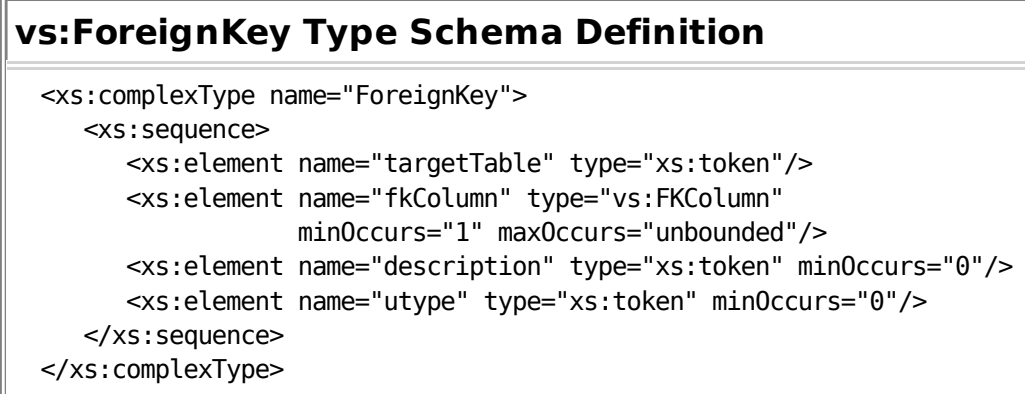

In this model, the source of the foreign key is the current table being described (i.e. represented by the <table> element that contains the vs: Foreignkey description, and thus doesn't need to be named explicitly). The key that is described points to the table given by the <targetTable> child element. Each child <fkcolumn> element then gives a pair of columns, one from the source table and one from the target table, that can be constrained to be equal in a query that joins the two tables.

\begin{tabular}{|l|ll|}
\hline \multicolumn{1}{|l|}{ vs:Foreign Key Metadata Elements } \\
\hline Element & \multicolumn{1}{c|}{ Definition } \\
\hline targetTable & $\begin{array}{ll}\text { Value type: } \\
\text { Semantic Meaning: }\end{array}$ & $\begin{array}{l}\text { string: xs: token } \\
\text { the fully-qualified name (including catalog and schema, } \\
\text { as applicable) of the table that can be joined with the } \\
\text { table containing this foreign key. } \\
\text { required }\end{array}$ \\
\hline fkColumn & Occurrences: & Value type: \\
\hline
\end{tabular}




\begin{tabular}{|c|c|c|}
\hline \multicolumn{3}{|c|}{ vs:ForeignKey Metadata Elements } \\
\hline Element & & Definition \\
\hline & $\begin{array}{l}\text { Semantic Mea } \\
\text { Occurrences: } \\
\text { Comments: }\end{array}$ & $\begin{array}{l}\text { a pair of column names, one from this table and one } \\
\text { from the target table that should be used to join the } \\
\text { tables in a query. } \\
\text { required; multiple occurrences are allowed. } \\
\text { There should be one <fkcolumn } \text { element for each column } \\
\text { that makes up the foreign key. }\end{array}$ \\
\hline description & $\begin{array}{l}\text { Value type: } \\
\text { Semantic Mea } \\
\text { Occurrences: }\end{array}$ & $\begin{array}{l}\text { string: xs: token } \\
\text { a free-text description of what this key points to and } \\
\text { what the relationship means } \\
\text { optional }\end{array}$ \\
\hline utype & $\begin{array}{l}\text { Value type: } \\
\text { Semantic Mea } \\
\text { Occurrences: } \\
\text { Comments: }\end{array}$ & $\begin{array}{l}\text { string: xs: token } \\
\text { : an identifier for a concept in a data model that the } \\
\text { association enabled by this key represents. } \\
\text { optional } \\
\text { The format defined in the VOTable standard, section } 4.1 \\
\text { [VOTable] is strongly recommended; see "Note on } \\
\text { UType Format" above. }\end{array}$ \\
\hline
\end{tabular}

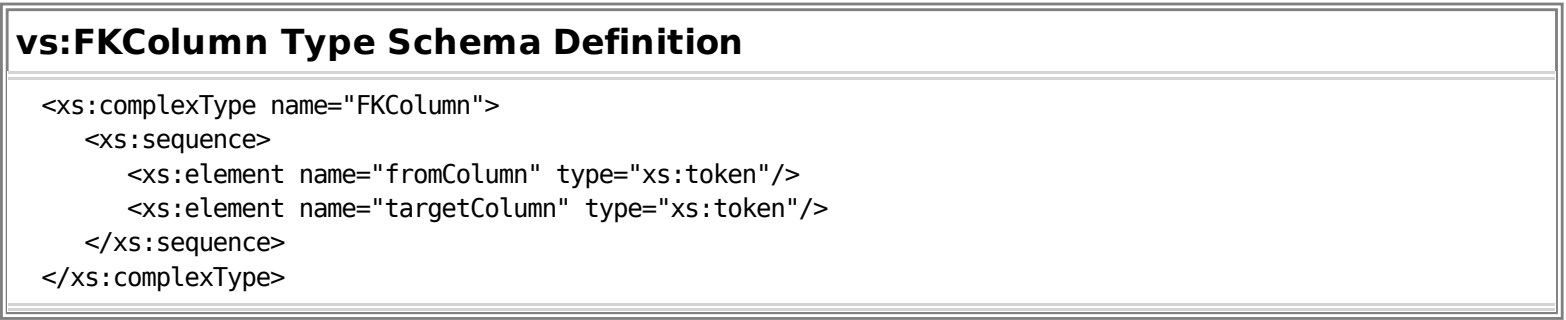

\begin{tabular}{|c|c|}
\hline \multicolumn{2}{|c|}{ vs:FKColumn Metadata Elements } \\
\hline Element & Definition \\
\hline fromColumn & 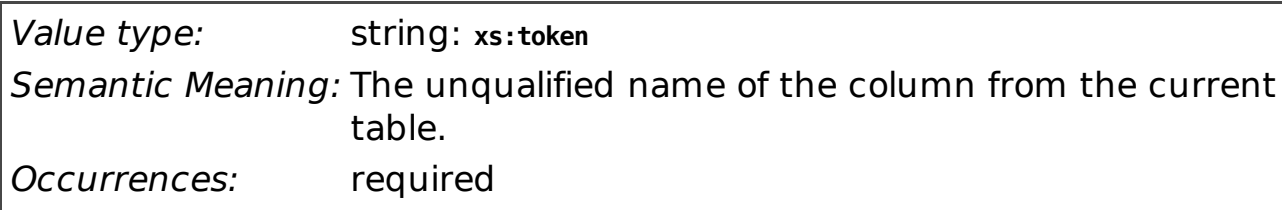 \\
\hline targetColumn & $\begin{array}{l}\begin{array}{l}\text { Value type: } \\
\text { Semantic Meaning: }\end{array} \\
\begin{array}{ll}\text { the unqualified name of the column from the target } \\
\text { table }\end{array} \\
\begin{array}{ll}\text { Occurrences: } & \text { required }\end{array}\end{array}$ \\
\hline
\end{tabular}

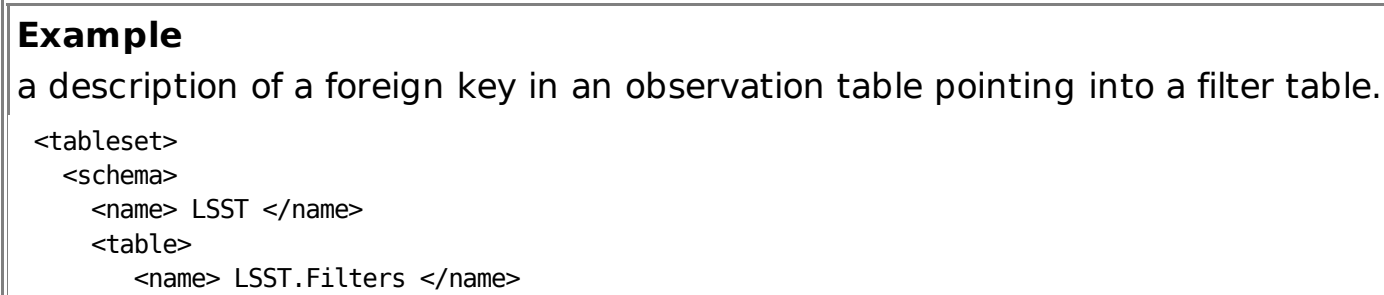




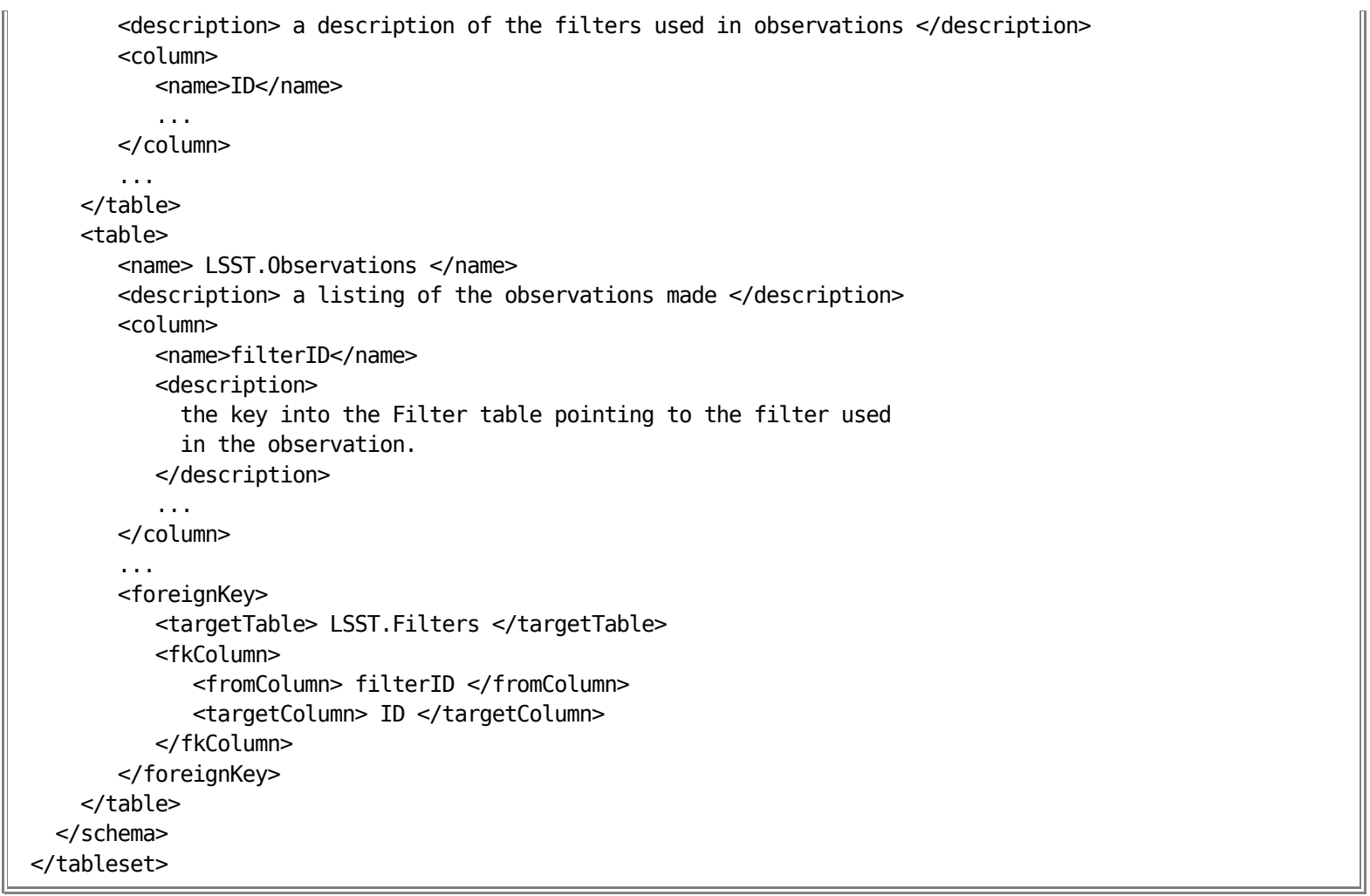

\subsubsection{Extending Table Metadata}

It is envisioned that it may be useful in the future to provide richer metadata for describing tables within a VOResource description than what are defined in this document. This document recommends the use of the following extension mechanisms when richer descriptions are desired:

1. Use extended types by applying the xsi:type attribute to the <tableset>, <schema>, <table>, $<$ column> and/or <dataType elements. The values provided in the attributes must refer to an XML type legally extended from the types associated with these elements according to the rules of XML Schema [schema] and the VOResource specification [VOR].

2. Apply a globally-defined attribute from a schema other than VODataService (i.e. from a namespace other than "http://www.ivoa.net/xml/VODataService/v1.1") to any of the <tableset>, <schema>, <table>, and/or <column> elements.

3. When the extended metadata is specific to how the table data is accessed via a particular service protocol, then the new metadata can be incorporated into a specific capability extension (as described in the VOResource specification [VOR]). This extension may make use of the various names within the <tableset> to indicate where the extension metadata apply.

4. Use the extendedType attribute of the <dataTypes element (see section 3.5.3) to indicate a more specific data type then those defined by the vs: TableParam type.

\subsection{Interface Type Extension: ParamHTTP}

The vs: ParamHTTP type is a specialized service interface description that extends the 
VOResource vr: Interface type (as recommended by [VOR], section 2.3.2). It describes a service interface that is invoke over HTTP via a GET or a POST [ are parameters encoded as name=value pairs, delimited by ampersands $(\&)$ and URL-encoded [ $\underline{\mathrm{URI}}]$. When the service is invoked as a GET service, this input list is appended to a base URL (where the result must form a legal URL. Usually, the URL contains a question mark (?) setting off a list of URL arguments to the URL:

\section{Example}

A service that takes 3 parameters: ra, dec, radius

http: //data . archive. edu/cgi-bin/search? ra=12.32\&dec=-10.3\&radius=0. 1

When the service is invoked as a POST, the encoded list of parameters are uploaded to the service as the HTTP Message Body.

\section{Example}

The above GET request example shown as an HTTP POST message.

POST /cgi-bin/search

User-Agent: Python script

Content-Type: application/x-www-form-urlencoded

Content-Lenth: 29

ra $=12.32 \& d e c=-10.3 \&$ radius $=0.1$

The vs:ParamHTtP type is intended for (but not limited to) use in describing an interface within a VOResource description of a service capability (as described in [VOR], section 2.2.2); that is, it can be invoked via the xsi:type attribute on an <interfaces element.

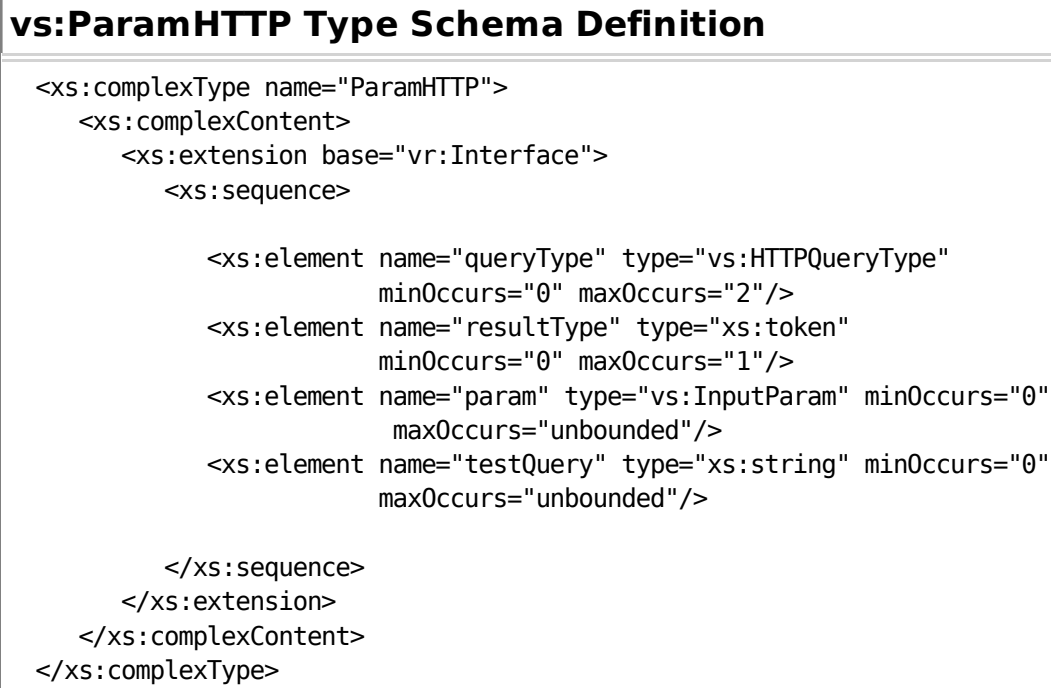

The extension metadata defined in the schema definition above are all optional. Nevertheless, even when an <interfaces instance does not include any of these extended child elements, the use of xsi: type="vs: ParamHTTP" indicates that the interface accessed via the URL given by the <accessURL> element complies with the general parameter-based protocol described in this section.

vs:ParamHTTP Extension Metadata Elements 


\begin{tabular}{|c|c|}
\hline Element & Definition \\
\hline queryType & $\begin{array}{ll}\begin{array}{ll}\text { Value type: } & \text { string with controlled values: vs:HTTPQueryType } \\
\text { Semantic Meaning: } & \text { The type of HTTP request supported by the interface, } \\
& \text { either GET or POST. }\end{array} \\
\begin{array}{ll}\text { Occurrences: } & \text { optional; } 2 \text { occurrences are allowed to indicate that both } \\
\text { GET and POST are supported }\end{array} \\
\begin{array}{ll}\text { Allowed Values: } & \text { GET or POST }\end{array}\end{array}$ \\
\hline resultType & \begin{tabular}{|ll} 
Value type: & a string in MIME type format: xs: token \\
Semantic Meaning: & The MIME type of a document returned in the HTTP \\
& response. \\
Occurrences: & optional
\end{tabular} \\
\hline param & 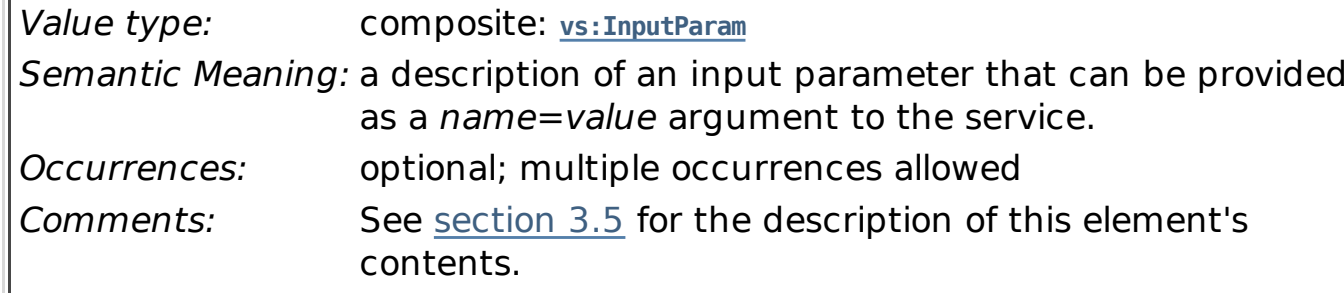 \\
\hline testQuery & $\begin{array}{ll}\text { Value type: } & \text { a string in MIME type format: xs: token } \\
\text { Semantic Meaning: an ampersand-delimited list of arguments that can be } & \\
& \text { used to test this service interface; when provided as the } \\
& \text { input to this interface, it will produce a legal, non-null } \\
& \text { response. }\end{array}$ \\
\hline
\end{tabular}

A important intended use of the vs:ParamHTTP type is describing the interface of an IVOA standard service protocol of the "simple" variety, such as the Simple Image Access Protocol [SIA]. In particular, it is recommended that specifications that define how a standard service is registered in a registry require the use of the vs:ParamHTTP interface type when it is applicable.

Normally, a VOResource description indicates its support for a standard protocol with <capability> element having a standardID attribute set to specific URI representing the standard. The standard will usually spell out the HTTP query type, the returned MIME type, and input parameters required for compliance; therefore, it is not necessary that the vs:ParamHTTP description provide any of the optional extended metadata, as they are already implied by the standardID. The description need only reflect the optional or locally unique features of the interface. In particular, description may include

- a <queryTypes element for a type that is not required by the standard (as long as the required query type is supported as well),

- <params elements for any optional parameters or local extended parameters (when allowed by the standard).

Of course, listing required parameters is always allowed, even when describing a standard 
interface as long as these are consistent with the service specification and the corresponding <param> elements include the attribute use="required" (see 3.5.1). The <param> elements for custom parameters that are not part of the standard (but are rather local customizations) should include the attribute std="false".

\subsection{Data Parameters}

The VODataService schema provides several element types for describing different kinds of data parameters used in datasets and services, including service input parameters and table columns. The parameter types allow one to fully describe a parameter in terms of metadata that includes name, data type, and meaning.

All the VODataService parameter types derive from a base type called vs:BaseParam which defines all the common parameter metadata except the data type.

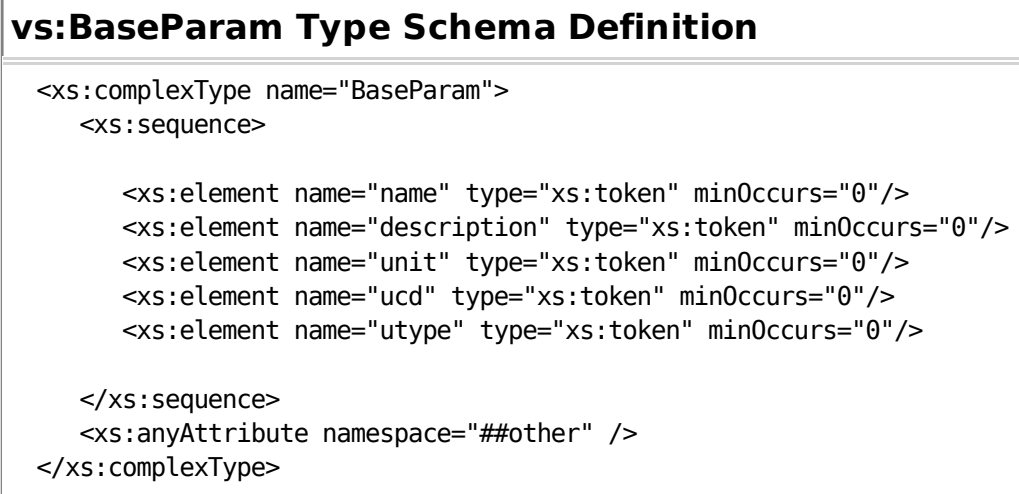

\begin{tabular}{|c|c|}
\hline \multicolumn{2}{|c|}{ vs:BaseParam Metadata Elements } \\
\hline Element & Definition \\
\hline name & $\begin{array}{ll}\text { Value type: } & \text { string: } x \text { : token } \\
\text { Semantic Meaning: } & \text { The name of the column. } \\
\text { Occurrences: } & \text { optional }\end{array}$ \\
\hline description & $\begin{array}{ll}\text { Value type: } & \text { string: xs: token } \\
\text { Semantic Meaning: a free-text description of the column's contents } \\
\text { Occurrences: } \quad \text { optional }\end{array}$ \\
\hline unit & $\begin{array}{ll}\text { Value type: } & \text { string: xs: token } \\
\text { Semantic Meaning: } & \text { the unit associated with all values associated with this } \\
& \text { parameter or table column. } \\
\text { Occurrences: } & \text { optional }\end{array}$ \\
\hline ucd & 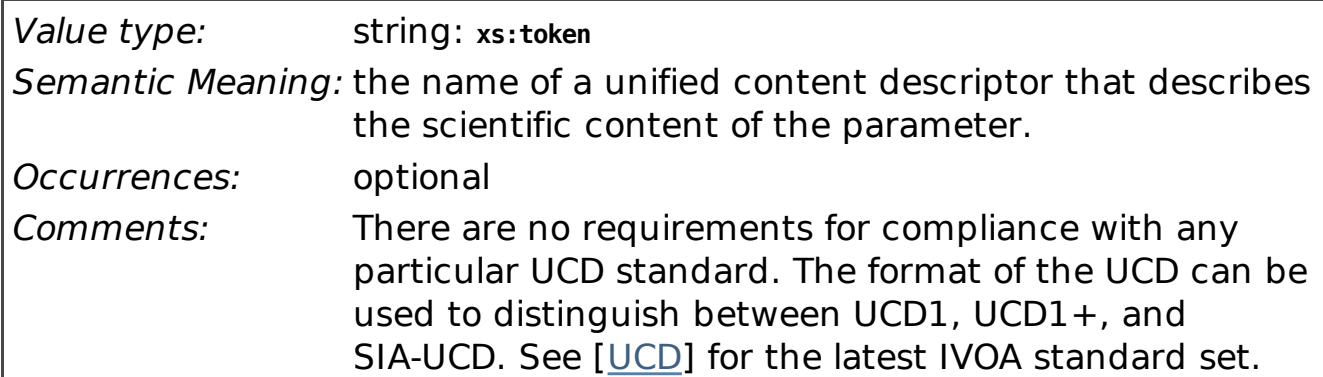 \\
\hline
\end{tabular}




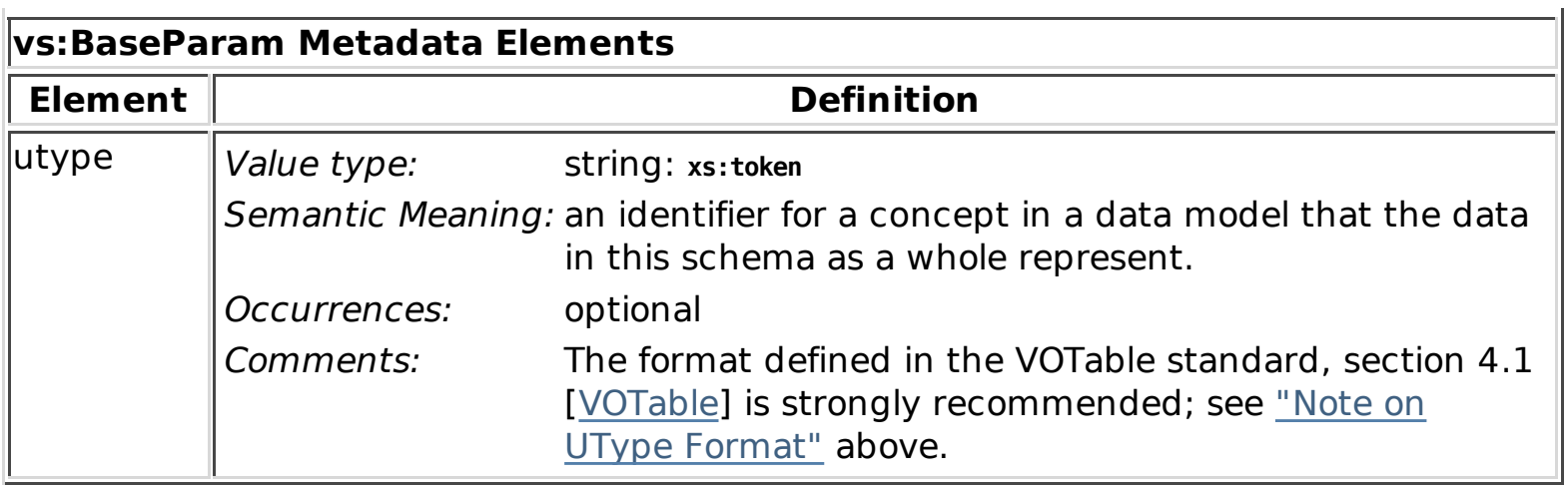

Leaving the data type metadatum out of vs:BaseParam allows the different kinds of parameters derived from vs:BaseParam to restrict the allowed data types to specific sets. The subsections below describe the different data types associated with input parameters (vs: InputParam) and table columns (vs:TableParam). The XML types associated with their <dataType> elements derive from a common parent, vs:DataType.

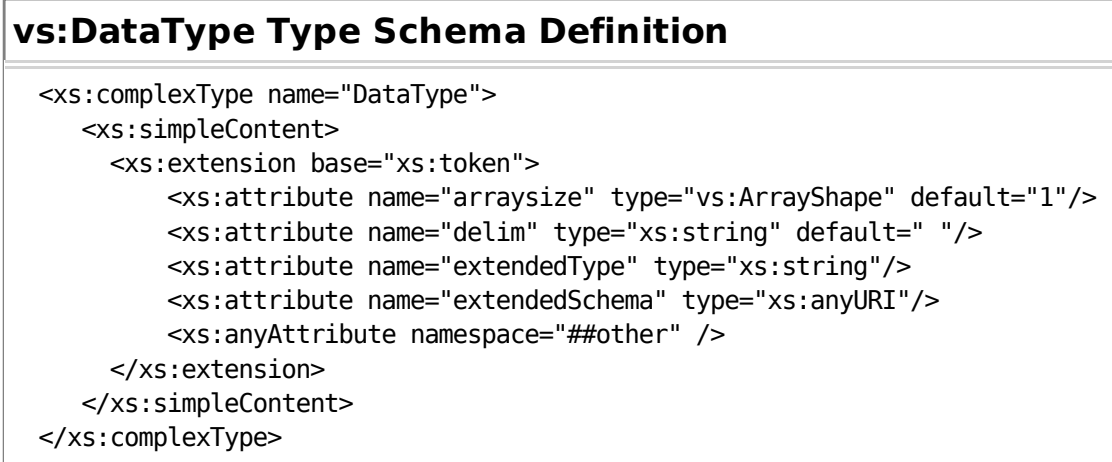

The content of an element of type vs:DataType is the name of the data type for the current parameter. When the element is explicitly a vs:DataType (as opposed to one of its derived types), there are no restrictions on the names that may be included.

A data type description can be augmented via a common set of vs:DataType attributes, defined below. The arraysize attribute indicates the parameter is an array of values of the named type. Its value describes the shape of the array, and the delim attribute may be used to indicate the delimiter that should appear between elements of an array value. Depending on the application context, these attribute may not be enough to effectively parse the array values, in which case more information must be brought to bear either through assumptions about a particular derived vs:DataType or through additional attributes.

More descriptive information about the type can be provided via extendedType and extendedSchema, which provide an alternate data type name. It's expected that this name will only be understood by a special subset of applications. The name given in the element content, then, represents a more commonly understood "fall-back" type. Arbitrary information can also be provided via any prefix-qualified, globally defined attribute drawn from an XML Schema other than VODataService (by virtue of the xs:anyAttribute specification shown above).

vs: DataType Attributes

\begin{tabular}{|ll}
\hline Attribute Definition &
\end{tabular}




\begin{tabular}{|c|c|c|}
\hline \multicolumn{3}{|c|}{ vs:DataType Attributes } \\
\hline $\mid$\begin{tabular}{|l|} 
Attribute \\
\end{tabular} & & Definition \\
\hline arraysize & $\begin{array}{l}\text { Value type: } \\
\text { Semantic Meaning: } \\
\text { Occurrences: } \\
\text { Default Value: }\end{array}$ & $\begin{array}{l}\text { the } \text { VOTable arraysize format (vs: ArrayShape): } L \times M x N \ldots \text {..., } \\
\text { where each } x \text {-delimited positive integer is a length along } \\
\text { a dimension of a multi-dimensional array. A single } \\
\text { integer indicates a one dimensional array. Instead of an } \\
\text { integer, the last length can be set to "*" which } \\
\text { indicates a variable length. } \\
\text { The attribute's presence indicates that parameter holds } \\
\text { an array values; the attribute's value indicates the } \\
\text { length of the array along each dimension of the multi- } \\
\text { dimensional array. } \\
\text { optional } \\
1 \text { (i.e. the parameter value is scalar) }\end{array}$ \\
\hline delim & $\begin{array}{l}\text { Value type: } \\
\text { Semantic Meaning: } \\
\text { Occurrences: } \\
\text { Comments: }\end{array}$ & $\begin{array}{l}\text { string: xs: string } \\
\text { the string that is used to delimit element of an array } \\
\text { value when arraysize is not "1". } \\
\text { optional. } \\
\text { Unless specifically disallowed by the context, } \\
\text { applications should allow optional spaces to appear in } \\
\text { an actual data value before and after the delimiter (e.g. } \\
\text { "1,5" when delim=","). }\end{array}$ \\
\hline extendedType & $\begin{array}{l}\text { Value type: } \\
\text { Semantic Meaning: } \\
\text { Occurrences: } \\
\text { Comments: }\end{array}$ & $\begin{array}{l}\text { string: xs: string. } \\
\text { The data value represented by this type can be } \\
\text { interpreted as of a custom type identified by the value } \\
\text { of this attribute. } \\
\text { optional } \\
\text { The name implies a particular expected format for the } \\
\text { data value that can be parsed into a value in memory. } \\
\text { If an application does not recognize this extendedType, } \\
\text { it should attempt to handle value assuming the type } \\
\text { given by the element's value. "string" (or its equivalent) } \\
\text { is a recommended default type. } \\
\text { This element may make use of the extendedSchema } \\
\text { attribute and/or any arbitrary (qualified) attribute to } \\
\text { refine the identification of the type. }\end{array}$ \\
\hline extendedSchema & $\begin{array}{l}\text { Value type: } \\
\text { Semantic Meaning: } \\
\text { Occurrences: } \\
\text { Comments: }\end{array}$ & $\begin{array}{l}\text { URI: xs: anyURI. } \\
\text { An identifier for the schema that the value given by the } \\
\text { extended attribute is drawn from. } \\
\text { optional } \\
\text { This attribute is normally ignored if the extended } \\
\text { element is not present. }\end{array}$ \\
\hline
\end{tabular}

Note that in the derived parameter description types described below, the <dataType> element is optional. Its absence from the parameter description does not mean that the parameter can support any data type; rather, it means that the data type simply has not 
been provided (which may limit what an application can do with the parameter). If a parameter can truly support any data type, the vs:BaseParam type can be used directly when the context permits.

\subsubsection{Input Parameters}

Actual parameters are normally described with types derived from vs:BaseParam. The vs:InputParam is intended for describing an input parameter to a service or function. The allowed data type names (given in the metadata table below) do not imply a size or precise format; rather, they are intended to be sufficient for describing an input paramter to a simple REST-like service or a function in a weakly-typed (e.g. scripting) language.

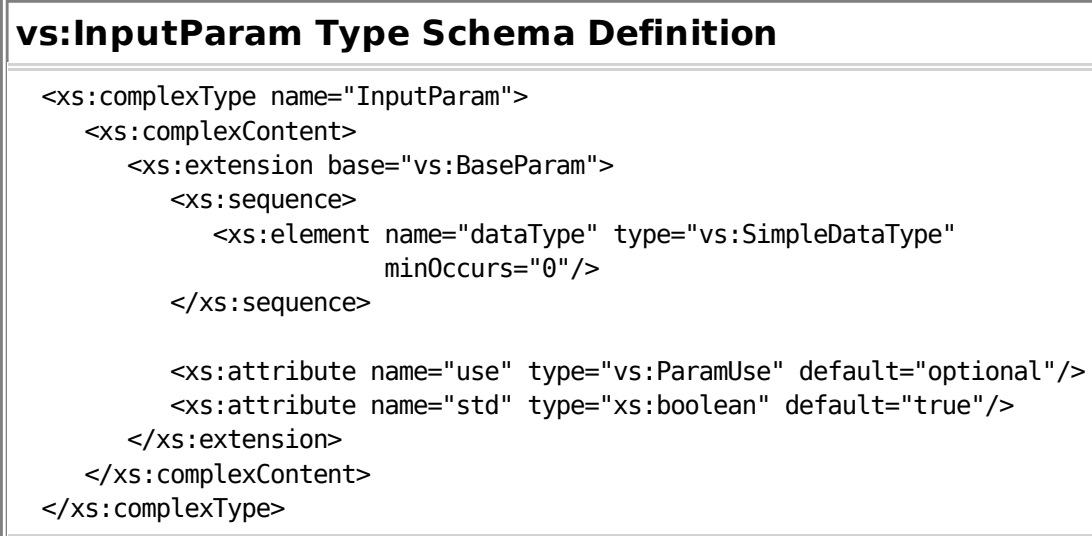

By fixing the <dataType> child element to that of the vs:SimpleDataType, the possible types are restricted to predefined set appropriate for input parameters.

\begin{tabular}{|c|c|c|}
\hline$\overline{\text { Element }}$ & & Definition \\
\hline dataType & $\begin{array}{l}\text { Value type: } \\
\text { Semantic Meaning: } \\
\text { Occurrences: } \\
\text { Allowed Values: }\end{array}$ & $\begin{array}{l}\text { string with optional attributes: vs:SimpleDataType } \\
\text { a type of data contained in the column. } \\
\text { optional } \\
\text { The following type names correspond to the same } \\
\text { data types defined in the VOTable standard [VOTable]: } \\
\text { boolean, char, integer, real, and complex. The additional } \\
\text { type, string, is equivalent to char when the attribute } \\
\text { arrayshape="*". }\end{array}$ \\
\hline
\end{tabular}

The vs: InputParam type accepts two attributes that indicate the role that the parameter plays as input to the service or function:

\begin{tabular}{|c|c|}
\hline vs:InputP & aram Attributes \\
\hline Attribute & Definition \\
\hline use & $\begin{array}{ll}\begin{array}{l}\text { Value type: } \\
\text { Semantic Meaning: }\end{array} & \text { An indication of whether this parameter is required to be } \\
& \text { provided for the application or service to work properly. } \\
\text { Occurrences: } & \text { optional }\end{array}$ \\
\hline
\end{tabular}




\begin{tabular}{|l||l|l|}
\hline Vs:InputParam Attributes \\
\hline Attribute & $\begin{array}{l}\text { Allowed Values: } \\
\text { std }\end{array}$ & $\begin{array}{l}\text { Dequired the parameter must be provided. } \\
\text { optional the parameter need not be provided (default). }\end{array}$ \\
\hline $\begin{array}{l}\text { Value type: } \\
\text { Semantic Meaning: }\end{array}$ & $\begin{array}{l}\text { true or false (xs:boolean) } \\
\text { reserved and defined by a standard interface. If false, it } \\
\text { represents an implementation-specific parameter that } \\
\text { effectively extends the behavior of the service or } \\
\text { application. The default is true. } \\
\text { optional }\end{array}$ \\
\hline
\end{tabular}

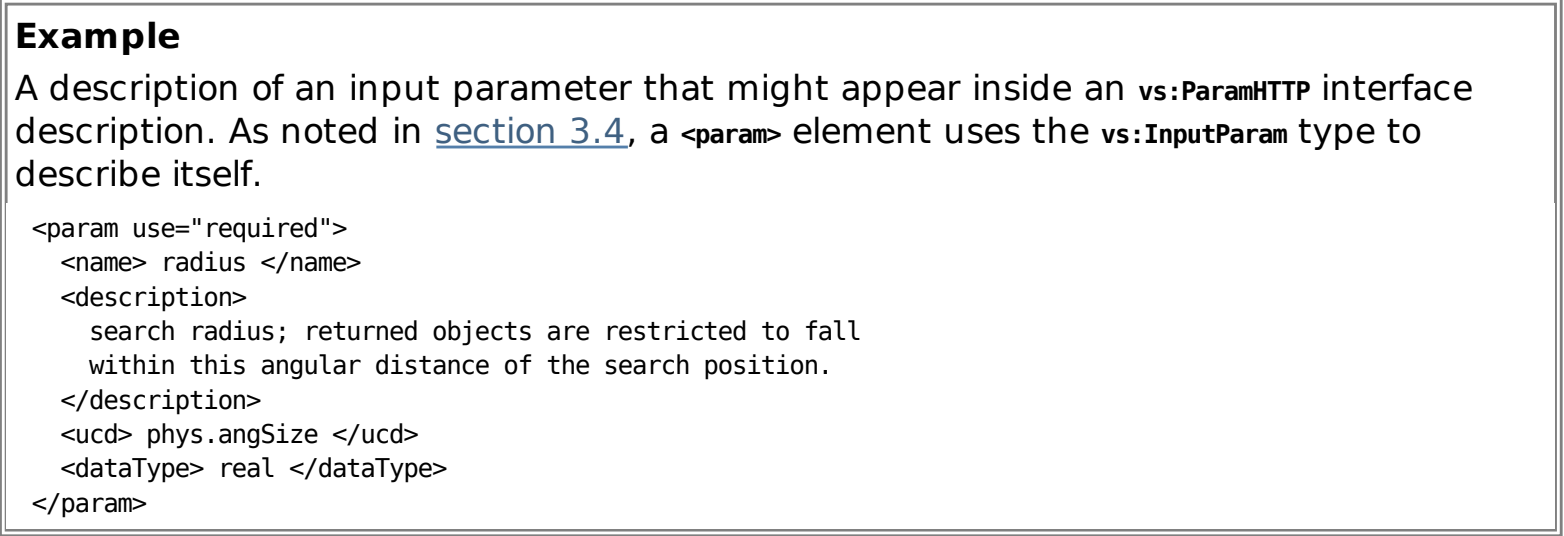

\subsubsection{Table Columns}

The vs: TableParam is also derived from vs:BaseParam, and is designed for describing a column of a table. 


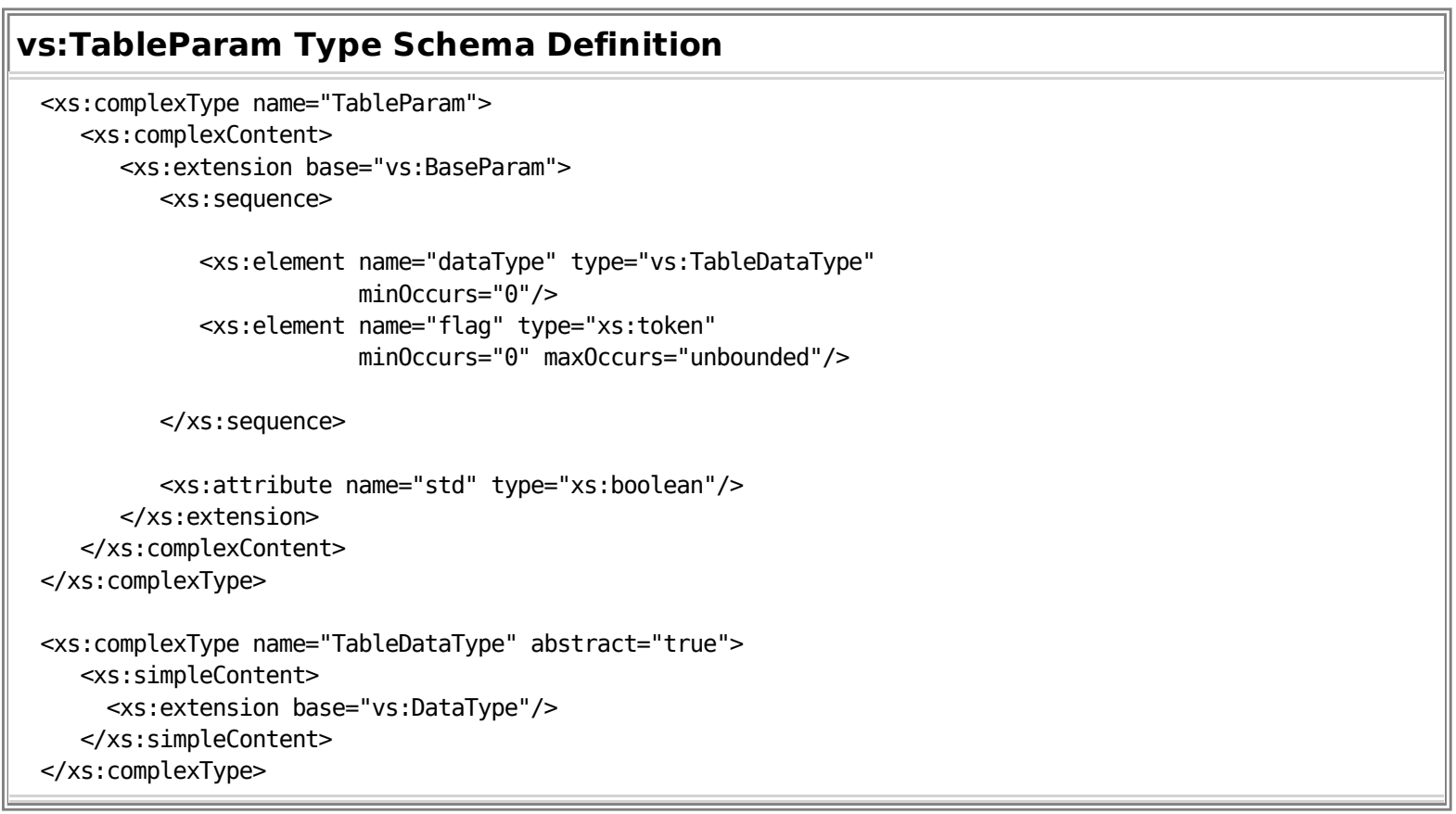

A table column's data type is indicated with the <dataType element with a name drawn from a standard set of names. The vs: Tableparam type is not restricted to a single standard set, and the VODataService schema defines two standard sets: one corresponding to VOTable data types [VOTable] and one for Table Access Protocol types. Because its XML type, vs: TableDataType is abstract, the <dataType> element MUST include an xsi:type attribute to indicate which standard set of type names is being used.

\section{Example}

A declination column called "Dec" is defined to have the VOTable-defined type, double $<$ column>

$<$ name $>$ Dec $<$ /name $>$

$<$ description $>$ the $\mathrm{J} 2000$ declination of the object </description $>$

$<$ ucd $>$ pos.eq. dec $</$ ucd $>$

$<$ dataType xsi:type="vs:V0TableType"> double </dataType >

$</$ column $>$

\begin{tabular}{|c|c|c|}
\hline \multicolumn{3}{|c|}{ vs:TableParam Extension Metadata Elements } \\
\hline Element & & Definition \\
\hline dataType & $\begin{array}{l}\text { Value type: } \\
\text { Semantic Meaning: } \\
\text { Occurrences: } \\
\text { Allowed Values: }\end{array}$ & $\begin{array}{l}\text { string with a required xsi: type attribute and additional } \\
\text { optional attributes: vs: TableDataType } \\
\text { a type of data contained in the column. } \\
\text { optional } \\
\text { The allowed type names are determined by value of } \\
\text { the xsi: type; see section } 3.5 .3 \text { below. }\end{array}$ \\
\hline flag & $\begin{array}{l}\text { Value type: } \\
\text { Semantic Meaning: } \\
\text { Occurrences: }\end{array}$ & $\begin{array}{l}\text { string with optional attributes: vs: TableDataType } \\
\text { a keyword representing traits of the column. } \\
\text { optional; multiple occurrences allowed }\end{array}$ \\
\hline
\end{tabular}




\begin{tabular}{|l|r|}
\hline vs:TableParam Extension Metadata Elements \\
\hline Element & Definition \\
\hline Recommended Values: indexed & $\begin{array}{l}\text { The column has an index on it for faster } \\
\text { search against its values. }\end{array}$ \\
primary & $\begin{array}{r}\text { The values column in the column represents } \\
\text { in total or in part a primary key for its table. } \\
\text { nutlable the column may contain null or empty values. } \\
\text { Other values are allowed. }\end{array}$ \\
\hline
\end{tabular}

\subsubsection{Table Column Data Types}

The VODataService schema defines two XML types that derive from vs: TableDataType: vs: VoTableType and vs: TAPType.

\begin{tabular}{|c|c|c|}
\hline \multicolumn{3}{|c|}{ Data Types derived from vs:TableDataType } \\
\hline $\begin{array}{l}\text { Value for } \\
\text { xsi:type }\end{array}$ & & Definition \\
\hline \multirow[t]{2}{*}{ vs:VOTableType } & Semantic Meani & $\begin{array}{l}\text { data types that correspond to the parameter and } \\
\text { column types defined in the VOTable schema } \\
\text { [VOTable]. }\end{array}$ \\
\hline & Allowed Values: & $\begin{array}{l}\text { boolean, bit, unsignedByte, short, int, long, char, unicodechar, } \\
\text { float, double, floatComplex, and doublecomplex. String } \\
\text { values of arbitrary length are represent by a value } \\
\text { of char with arraysize="*" }\end{array}$ \\
\hline \multirow[t]{2}{*}{ vs:TAPType } & Semantic Meani & $\begin{array}{l}\text { : data types that correspond column types defined in } \\
\text { the Table Access Protocol (v1.0) [TAP]. }\end{array}$ \\
\hline & Allowed Values: & $\begin{array}{l}\text { BOOLEAN, SMALLINT, INTEGER, BIGINT, REAL, DOUBLE, TIMESTAMP, CHAR, } \\
\text { VARCHAR, BINARY, VARBINARY, POINT, REGION, CLOB, and BLOB. } \\
\text { String values are represented via VARCHAR. }\end{array}$ \\
\hline
\end{tabular}

The vs:TAPType XML type provides an additional attribute, size, corresponding to the "size" column from the TAP_SCHEMA.columns defined by TAP:

\begin{tabular}{|c|c|}
\hline \multicolumn{2}{|c|}{ Additional Attribute for the vs:TAPType set of column data types } \\
\hline Attribute & Definition \\
\hline size & $\begin{array}{ll}\text { Value type: } & \text { a positive integer: xs: positiveInteger } \\
\text { Semantic Meaning: } & \text { The length of the variable-length data type. } \\
\text { Occurrences: } & \text { optional } \\
\text { Comments: } & \text { In the context of TAP, this attribute is only meaning } \\
& \text { when the data type is CHAR or BINARY; see discussion below. }\end{array}$ \\
\hline
\end{tabular}

\section{Example}

a representation of a string type using the vs: voTableType set of types:

$<$ column>

$<$ name $>$ id $<$ /name $>$

$<$ description $>$ the object identifier </description $>$

$<$ ucd $>$ meta.id </ucd $>$ 
$<$ dataType xsi:type="vs:VOTableType" arraysize=" $*$ " $>$ char $<$ /dataType $>$

$<$ column $>$

the same column described using the vs: TAPType set of types:

$<$ column $>$

$<$ name $>$ id $<$ /name $>$

$<$ description $>$ the object identifier </description>

$<$ ucd $>$ meta.id $<$ /ucd $>$

$<$ dataType xsi:type="vs:TAPType"> VARCHAR </dataType $>$

$<$ column $>$

the same column again described using the vs: TAPType set of types, assuming a fixedlength string:

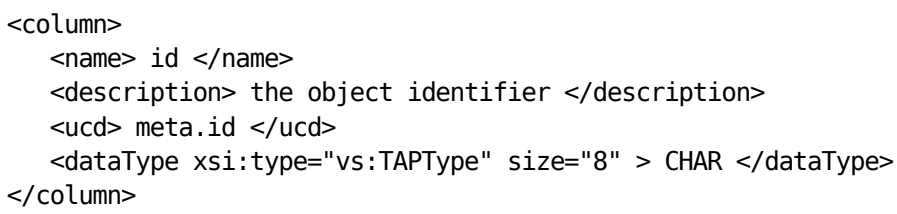

In general, the vs:TableParam's <dataType> can support any non-abstract type legally derived from vs: TableDataType. However, in the context of a vs:Datacollection or vs:CatalogService resource description, it is strongly recommended that either vs:voTableType or vs: TAPType (or some other IVOA standard type derived from vs: TableDataType) be used to ensure maximum interoperability. When the actual column type is not well matched to a type from one of these standard sets, authors are encouraged to use the extendedType attribute to refer to a more specific type. Note that the TAP standard [TAP] defines an explicit mapping between TAP_SCHEMA types and VOTable types. Thus, in the context of a vs: Catalogservice resource description that supports a TAP capability (perhaps in addition to other catalog services like Simple Cone Search [SCS]), use of the vs: TAPType data type is preferred.

Note:

The motivation for providing two standard data type sets, vs:v0TableType and vs:TAPType, is to maximize the ease of generating the table description, particular as part of the VO Standard Interface [VOSI] and for legacy services. The table description for "stand-alone" SIA, SCS, and SSA services can be readily generated using the vs:voTableType data types from these interface's respective metadata queries. Newer services supporting TAP could generate its description using its TAP_SCHEMA queries.

The motivation for specifying a column's data type using the xsi:type mechanism is mainly to allow for the possibility that the official TAP data types will evolve. This allows the IVOA to define new data type sets without updating the VODataService standard. Using non-IVOAstandardized data type names is expected to undermine interoperability and so is therefore discouraged.

\section{Appendix A: The VODataService XML Schema}

\section{The Complete VOResource Schema}

$<$ ?xml version="1.0" encoding="UTF-8"?>

<xs: schema targetNamespace="http://www.ivoa.net/xml/V0DataService/v1.1" xmlns:xs="http://www.w3.org/2001/XMLSchema" 


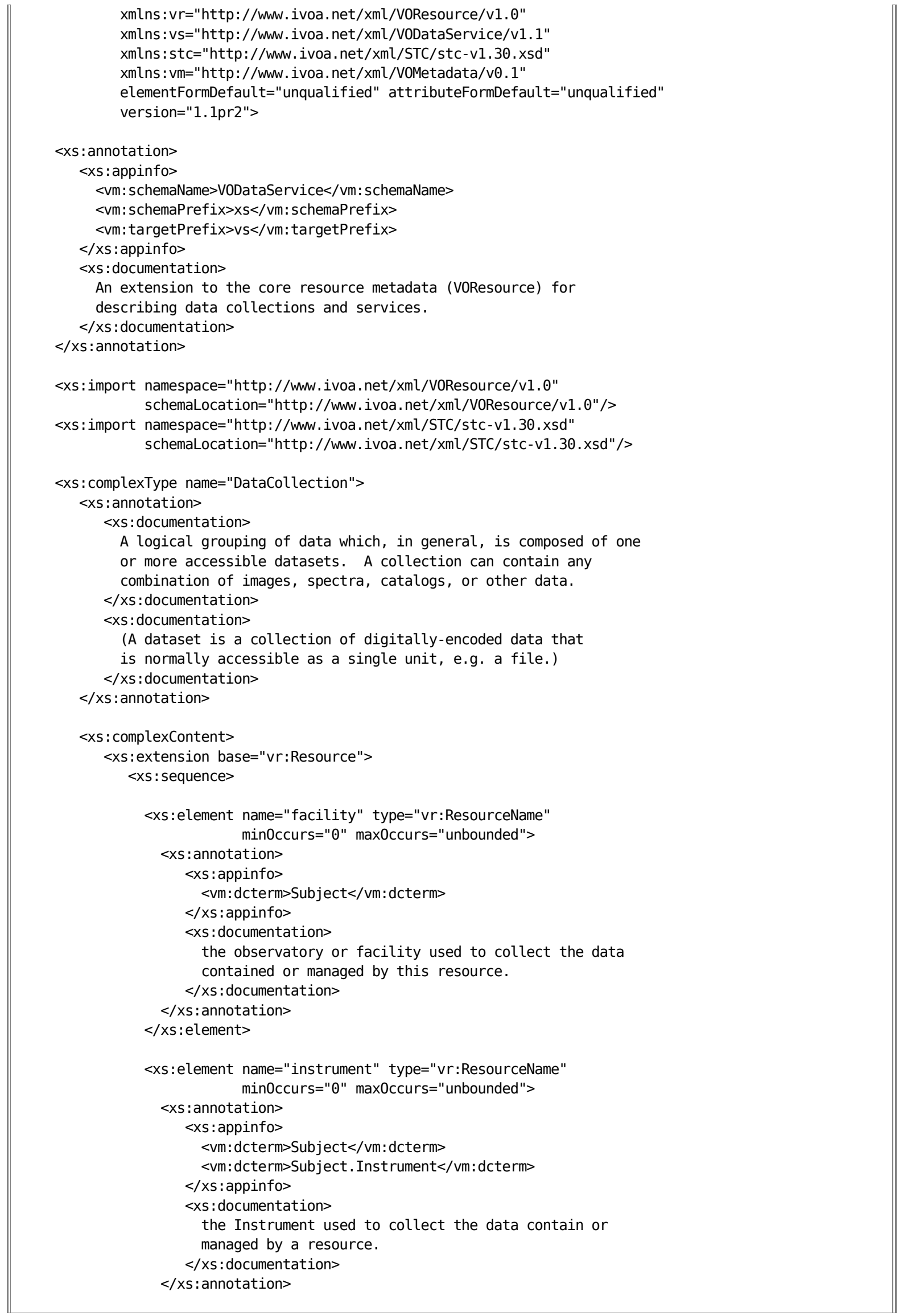




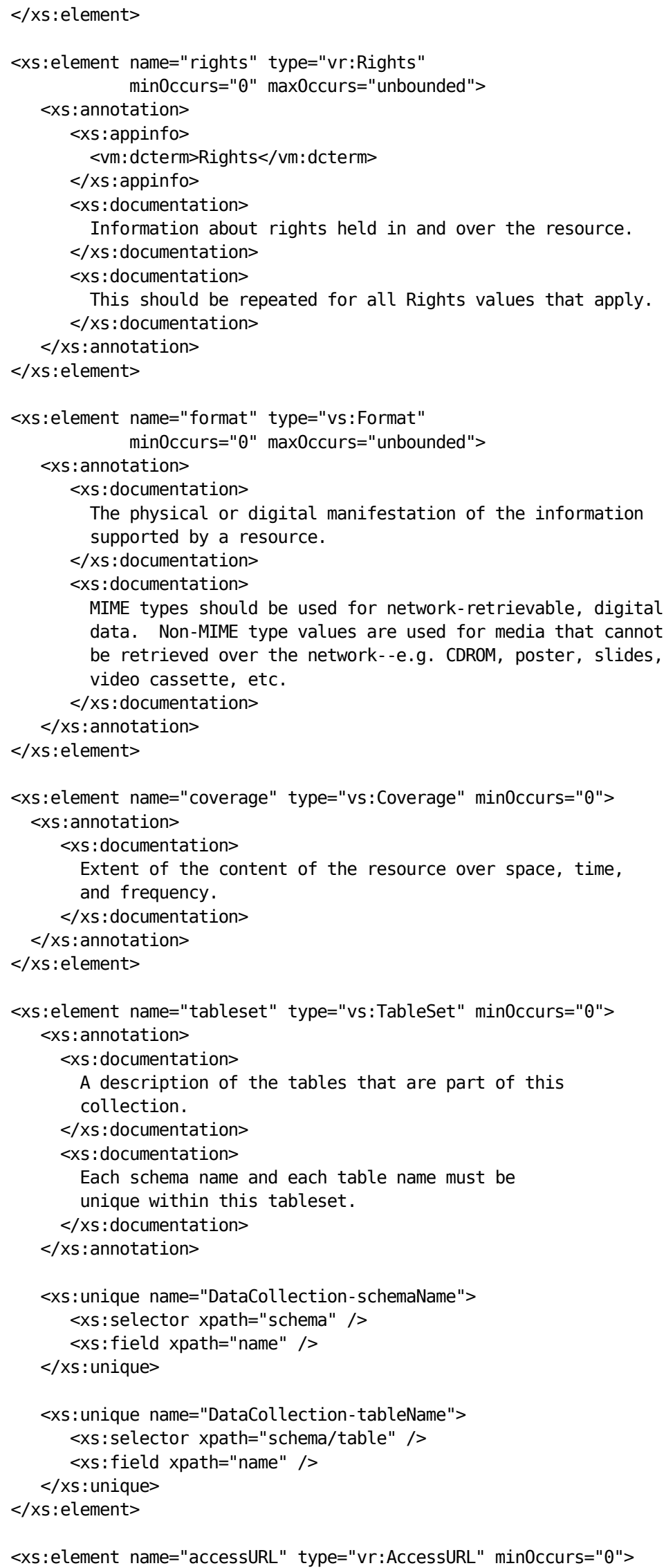




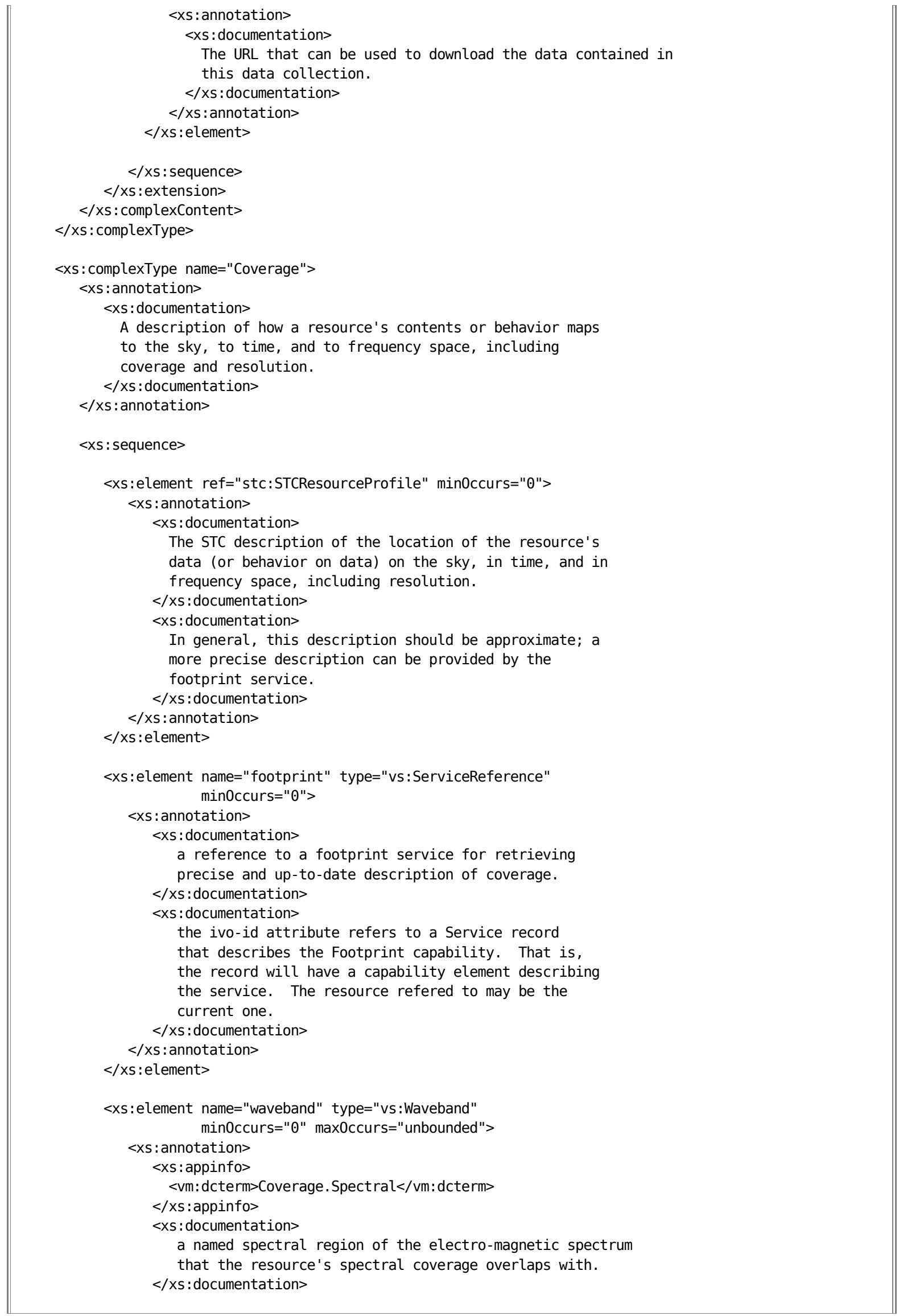




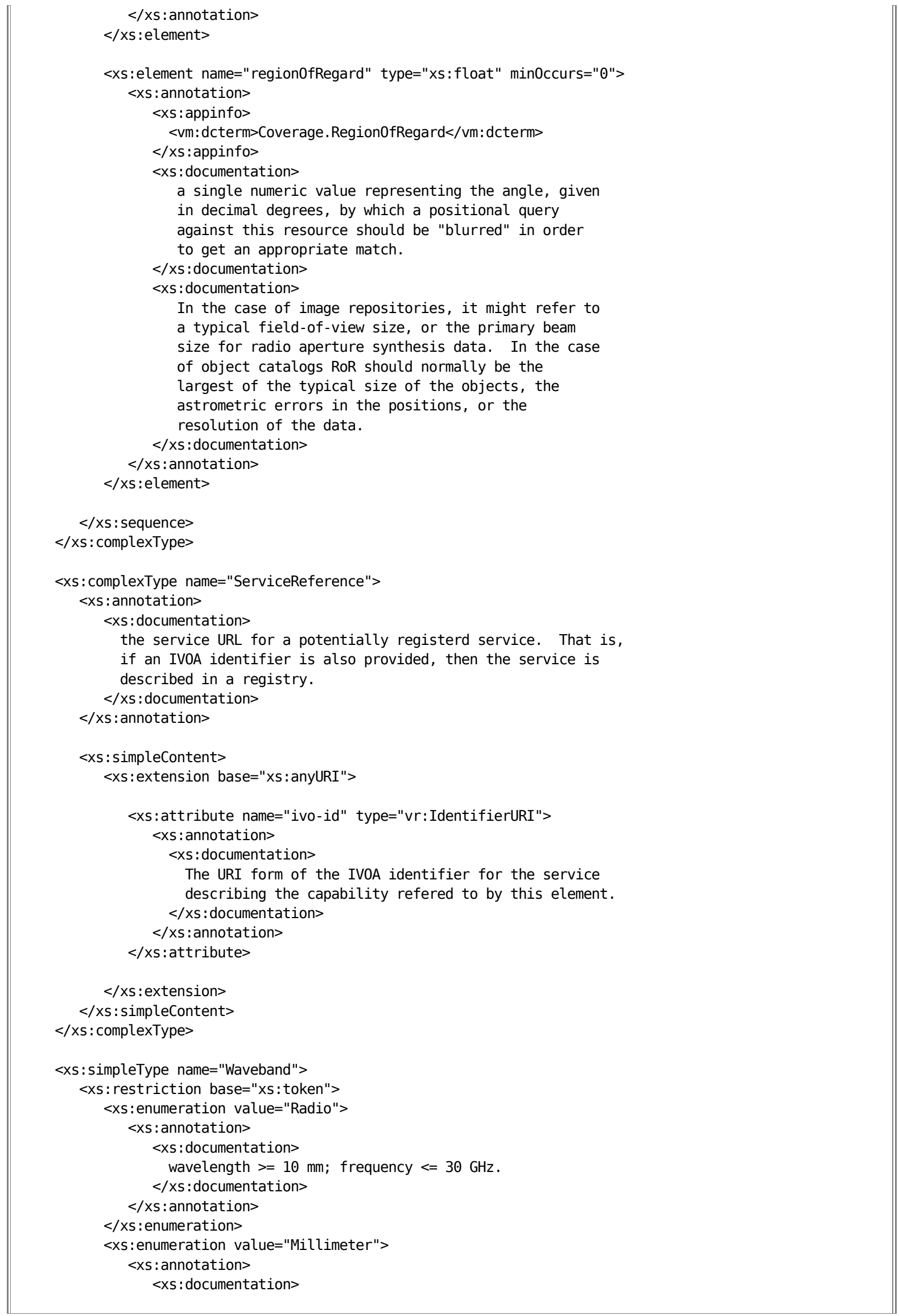




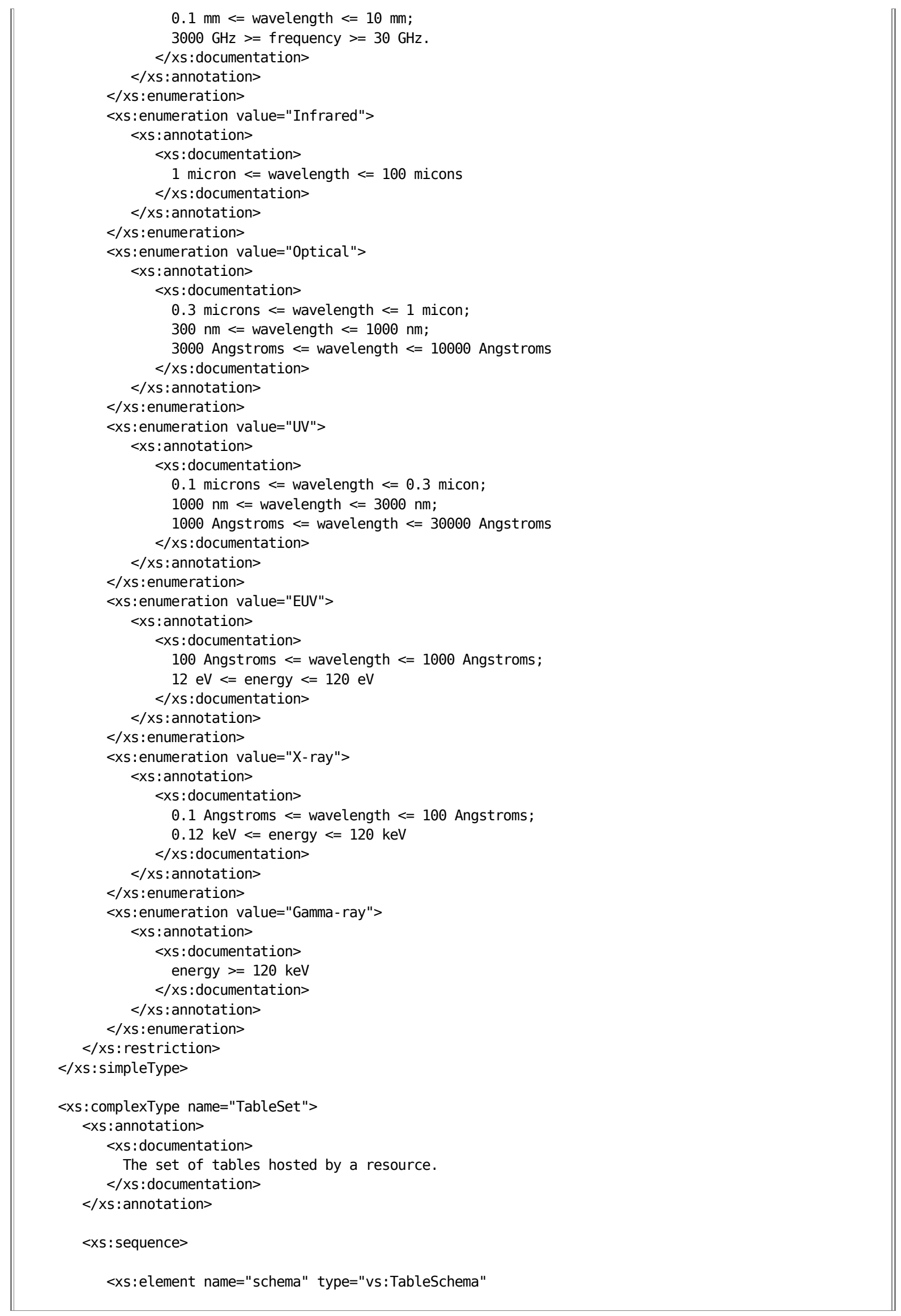




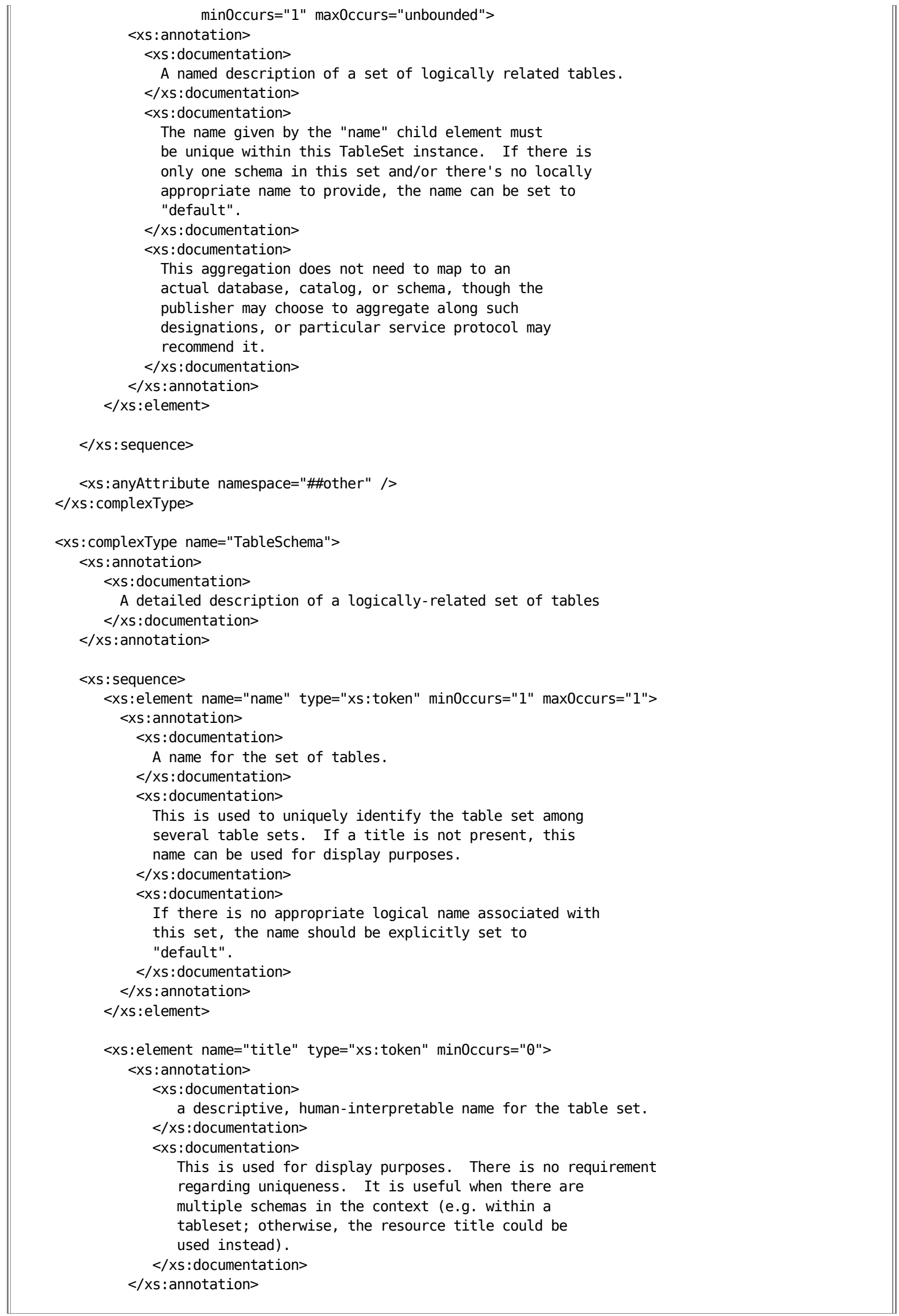




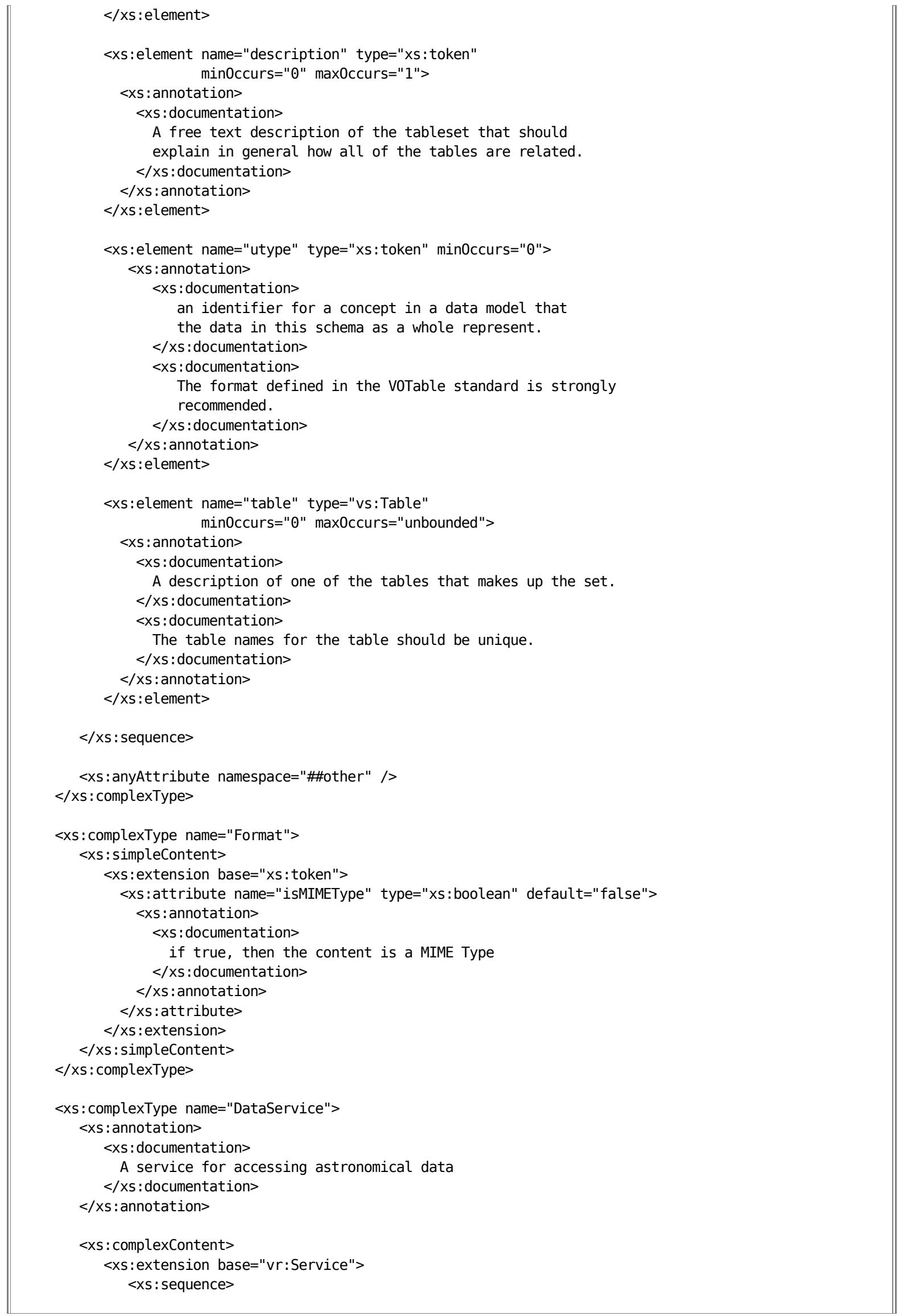




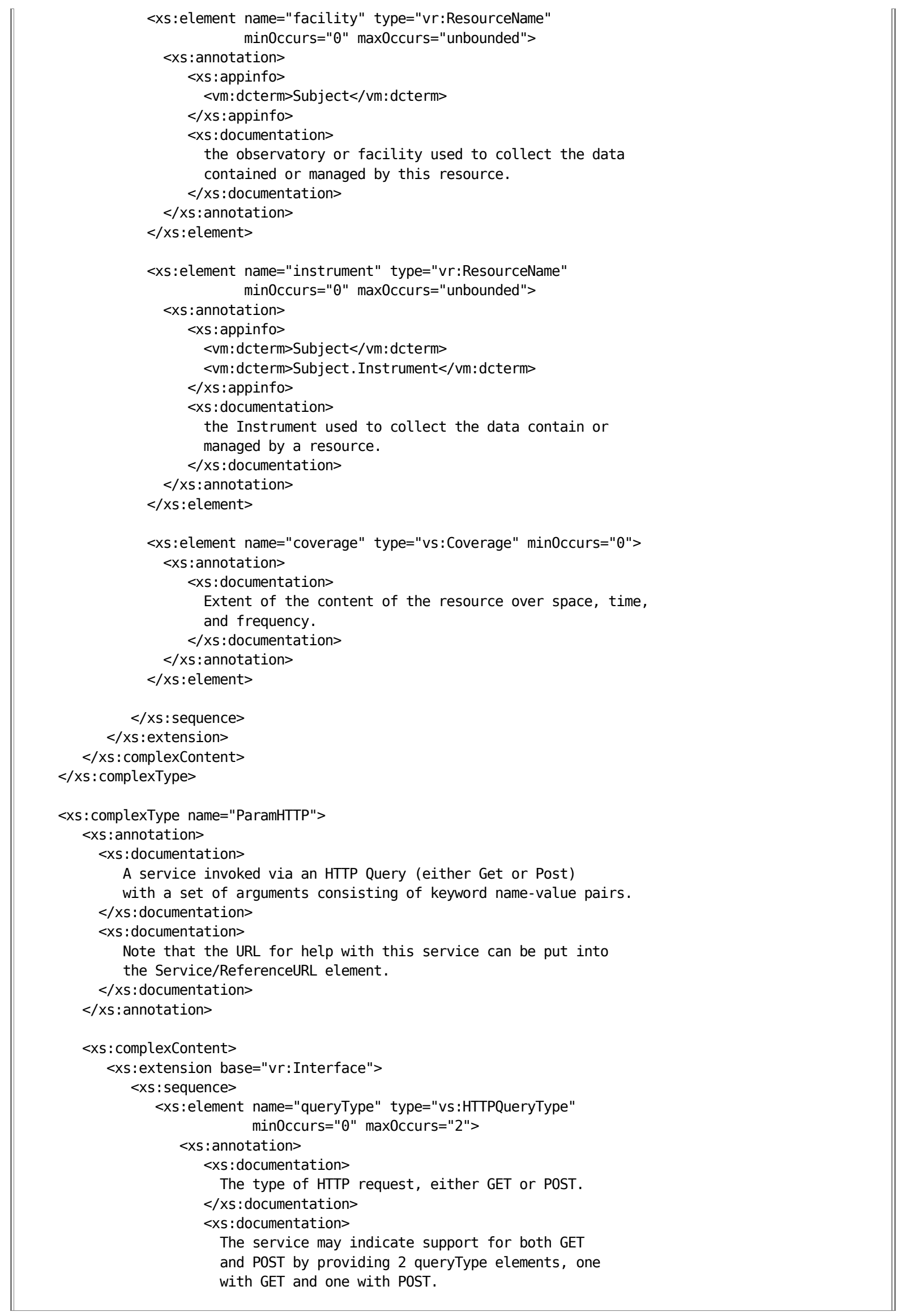




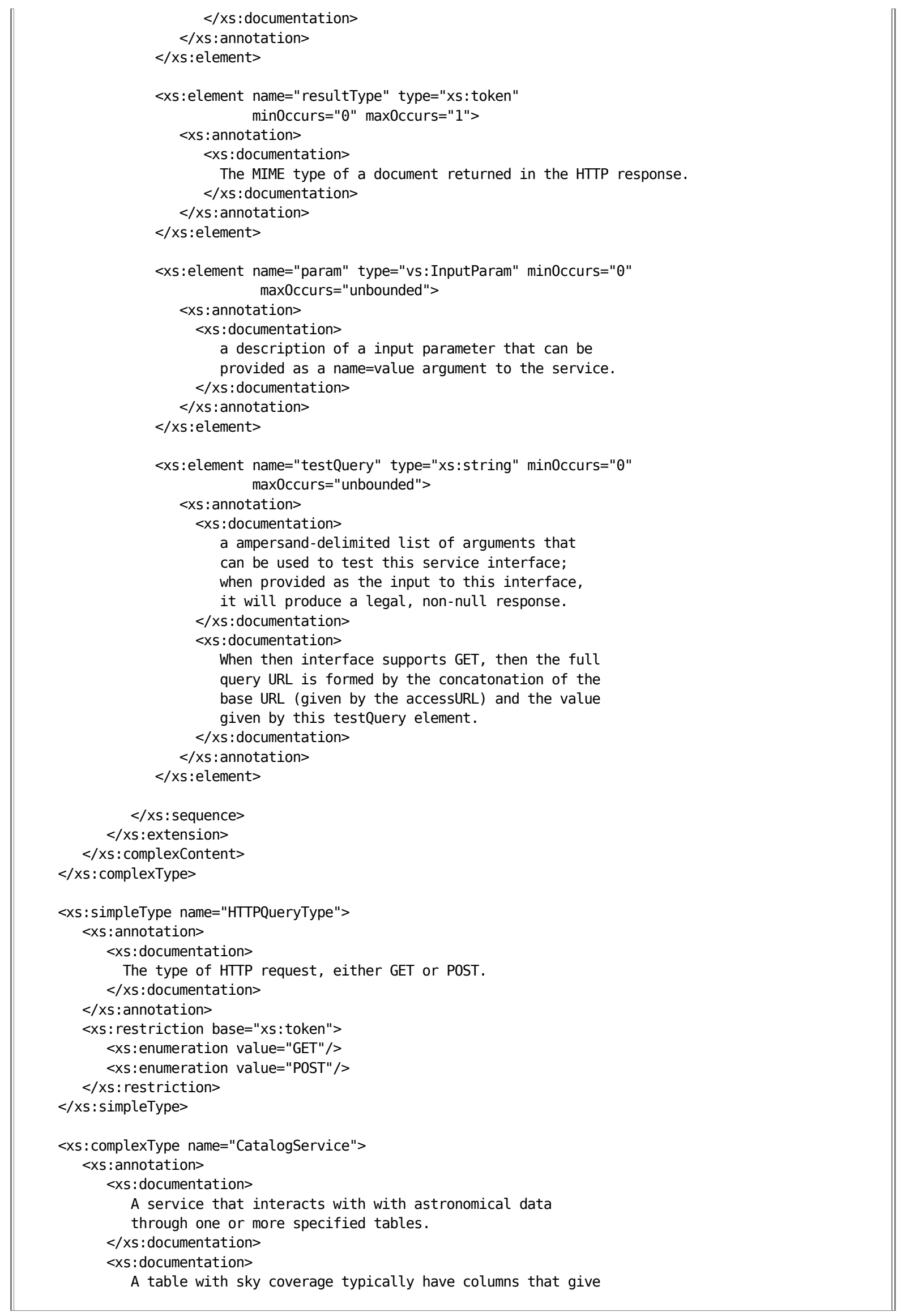




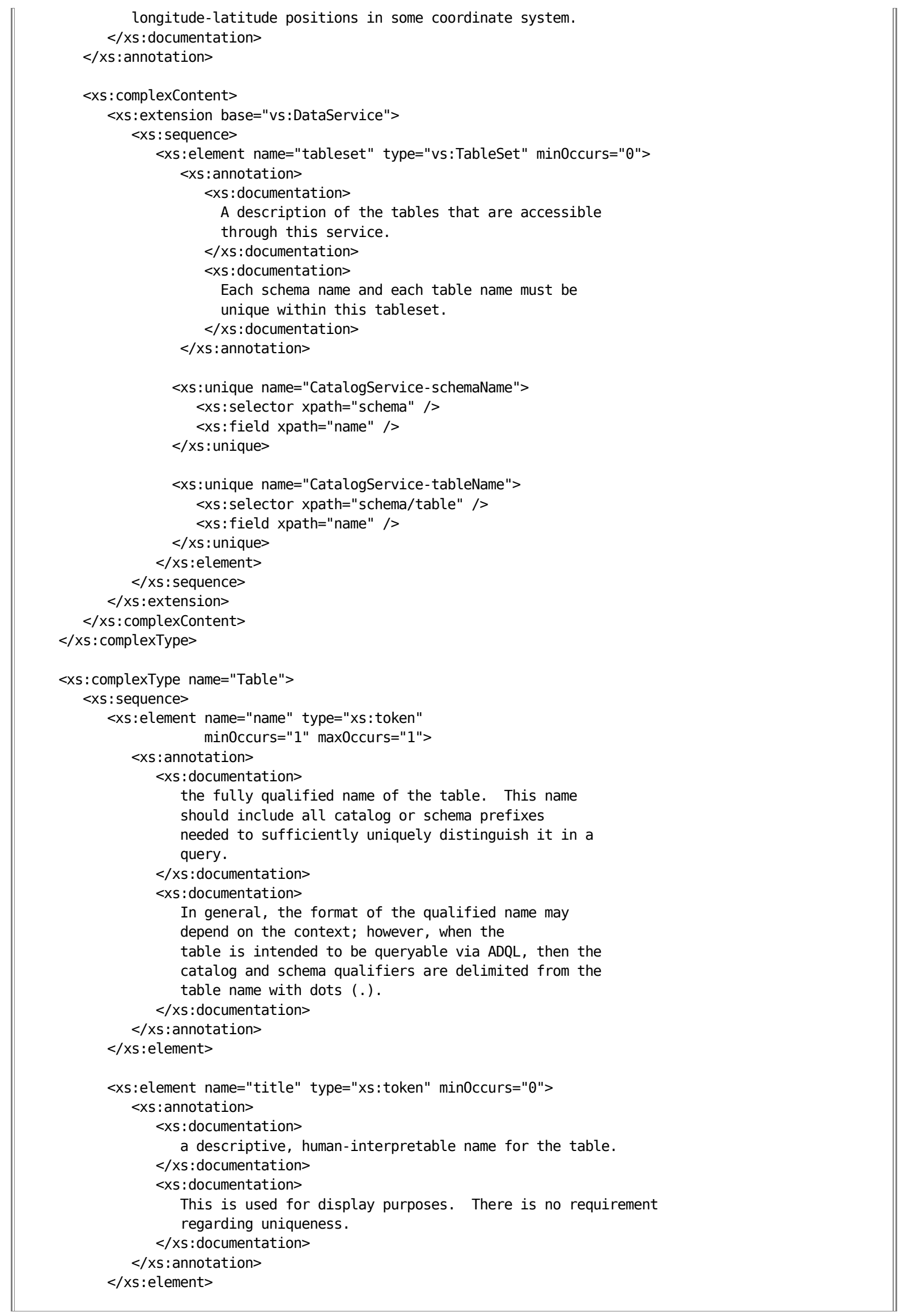


$<x s$ : element name="description" type="xs: token" min0ccurs=" 0 "> $<x$ : annotation>

$<x s$ : documentation>

a free-text description of the table's contents

$</ x s$ :documentation $>$

$</ x s$ :annotation $>$

$</ x s$ : element $>$

$<x s$ : element name="utype" type="xs: token" min0ccurs=" 0 ">

$<x$ : annotation>

$<x s$ : documentation>

an identifier for a concept in a data model that

the data in this table represent.

$</ x s$ : documentation $>$

$<x s$ : documentation>

The format defined in the voTable standard is highly

recommended.

$</ x s$ : documentation $>$

$</ x s$ :annotation $>$

$</ x s$ : element $>$

$<x s$ : element name="column" type="vs:TableParam"

min0ccurs=" 0 " max0ccurs="unbounded" >

$<x$ : annotation $>$

$<x s$ : documentation $>$

a description of a table column.

$</ x s$ : documentation>

$</ x s$ :annotation>

$</ x s$ : element $>$

<xs: element name="foreignKey" type="vs: ForeignKey" min0ccurs=" 0 " max0ccurs="unbounded" >

$<x$ : annotation>

$<x s$ : documentation>

a description of a foreign keys, one or more columns from the current table that can be used to join with another table.

$</ x s$ :documentation $>$

$</ x s$ :annotation $>$

$</ x s$ : element $>$

$</ x s$ : sequence $>$

$<x s$ :attribute name="type" type="xs: string">

$<x$ : annotation>

$<x$ : documentation>

a name for the role this table plays. Recognized

values include "output", indicating this table is output

from a query; "base table", indicating a table

whose records represent the main subjects of its

schema; and "view", indicating that the table represents

a useful combination or subset of other tables. Other

values are allowed.

$</ x s$ : documentation $>$

$</ x s$ :annotation $>$

$</ x s$ :attribute $>$

$<x s$ :anyAttribute namespace="\#\#ther" />

$</ x s$ : complexType>

$<x s$ : complexType name="BaseParam">

$<x$ : annotation>

$<x s$ : documentation>

a description of a parameter that places no restriction on

the parameter's data type. 


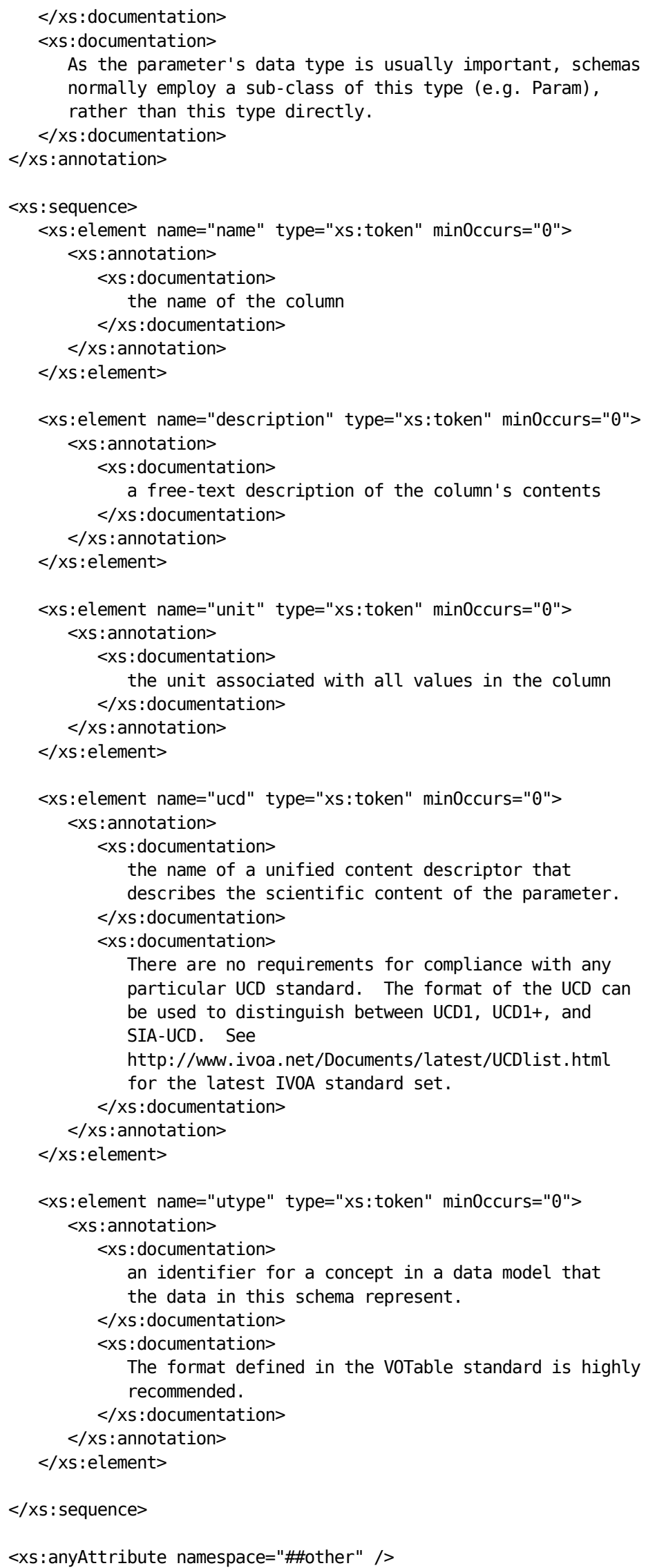




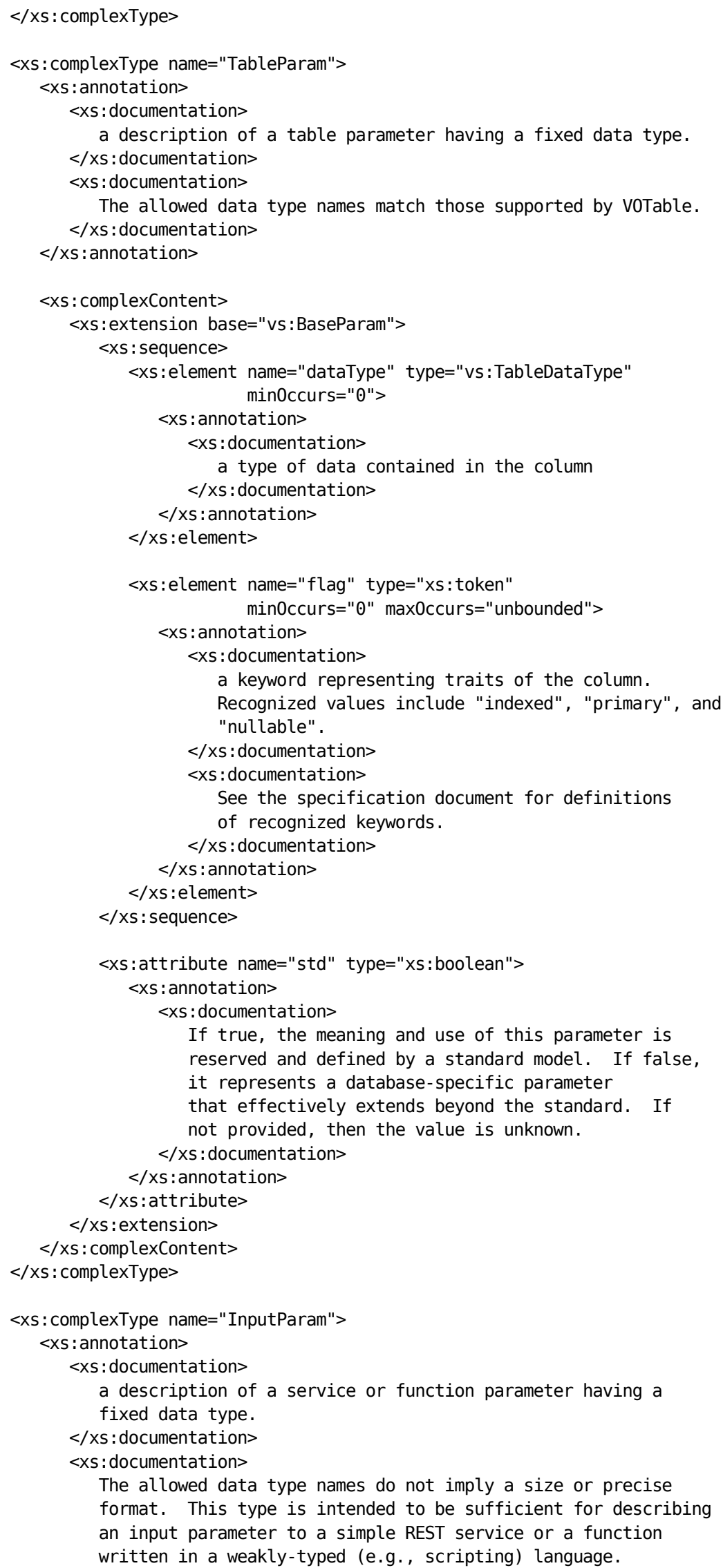




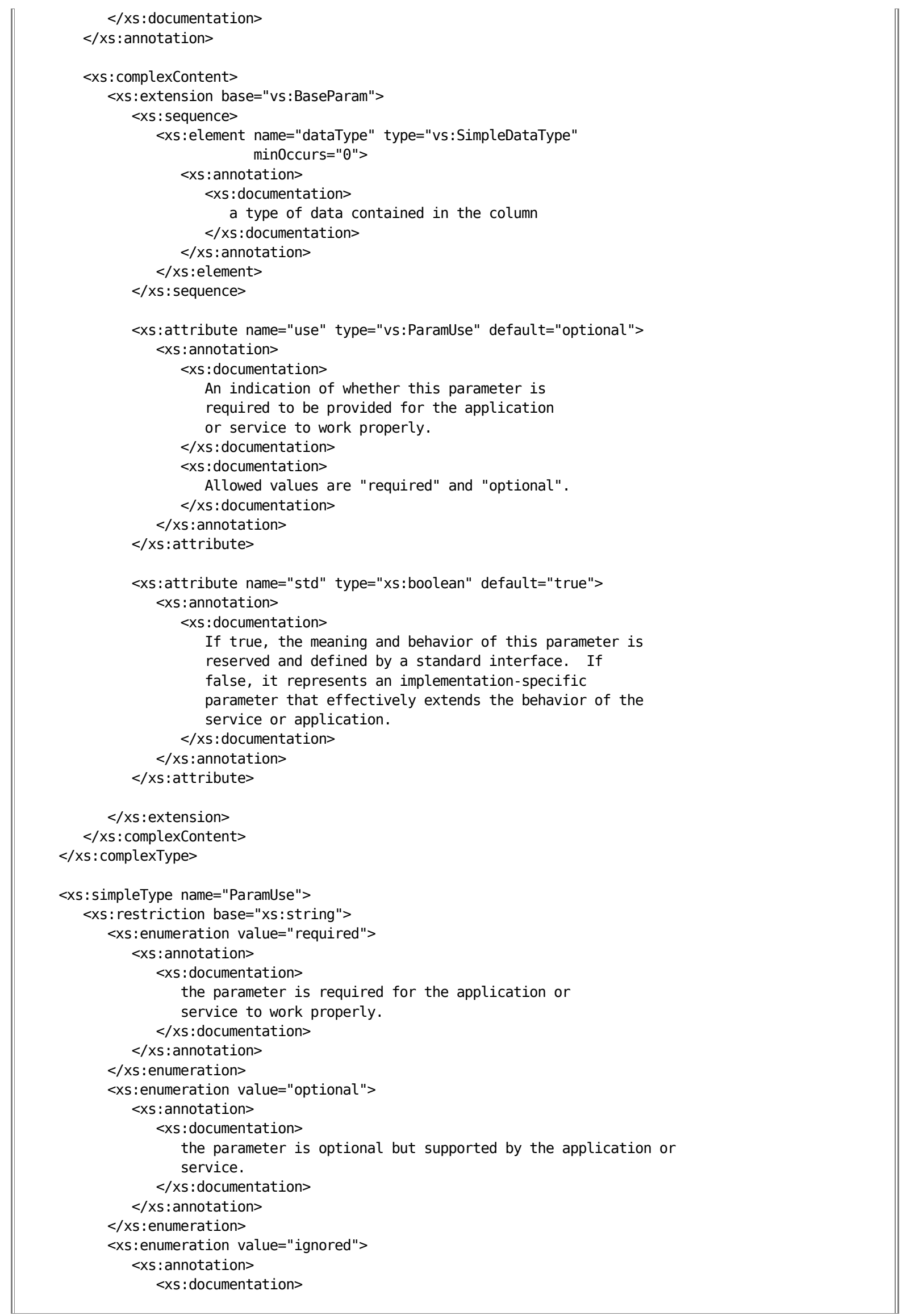




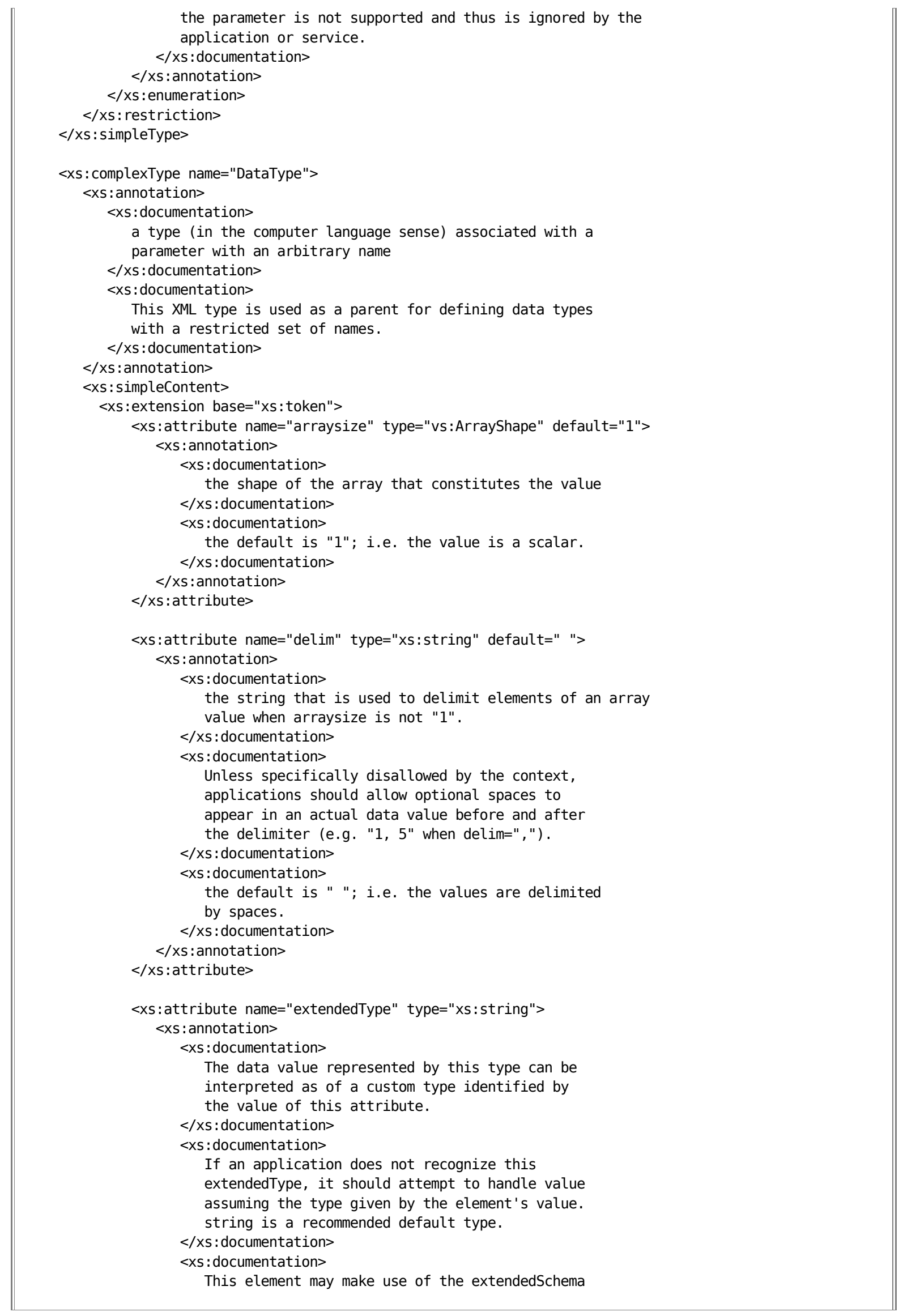




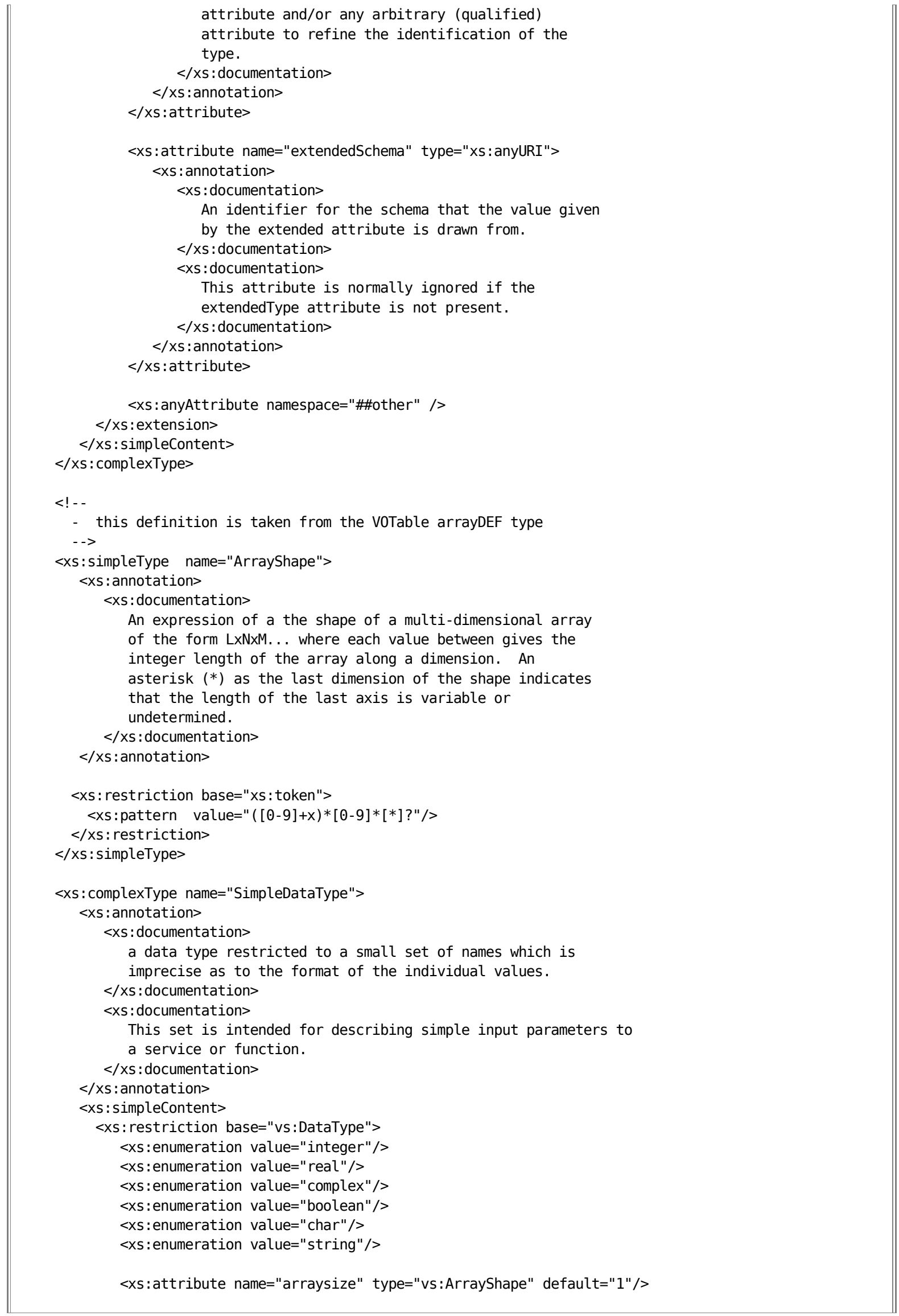




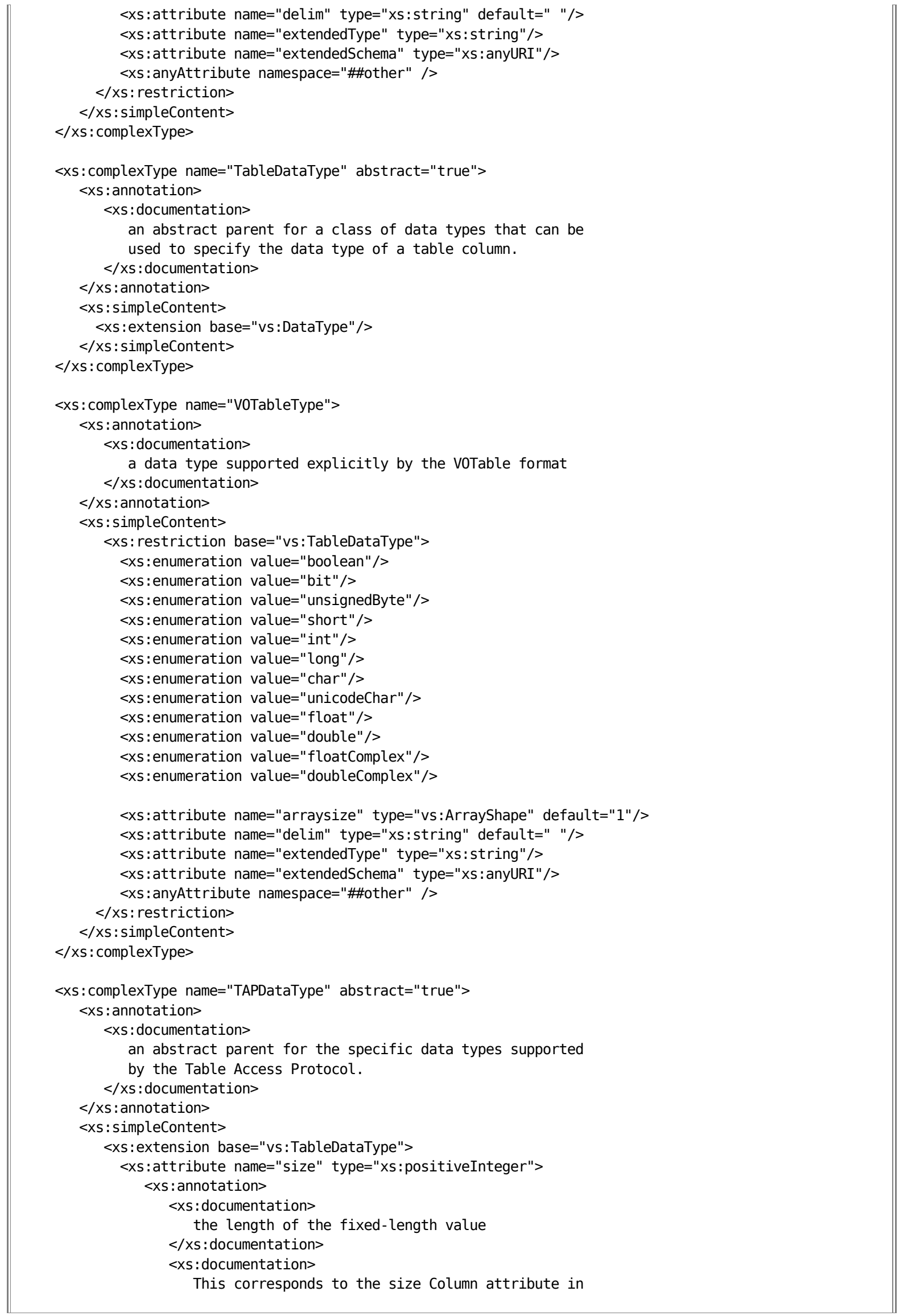




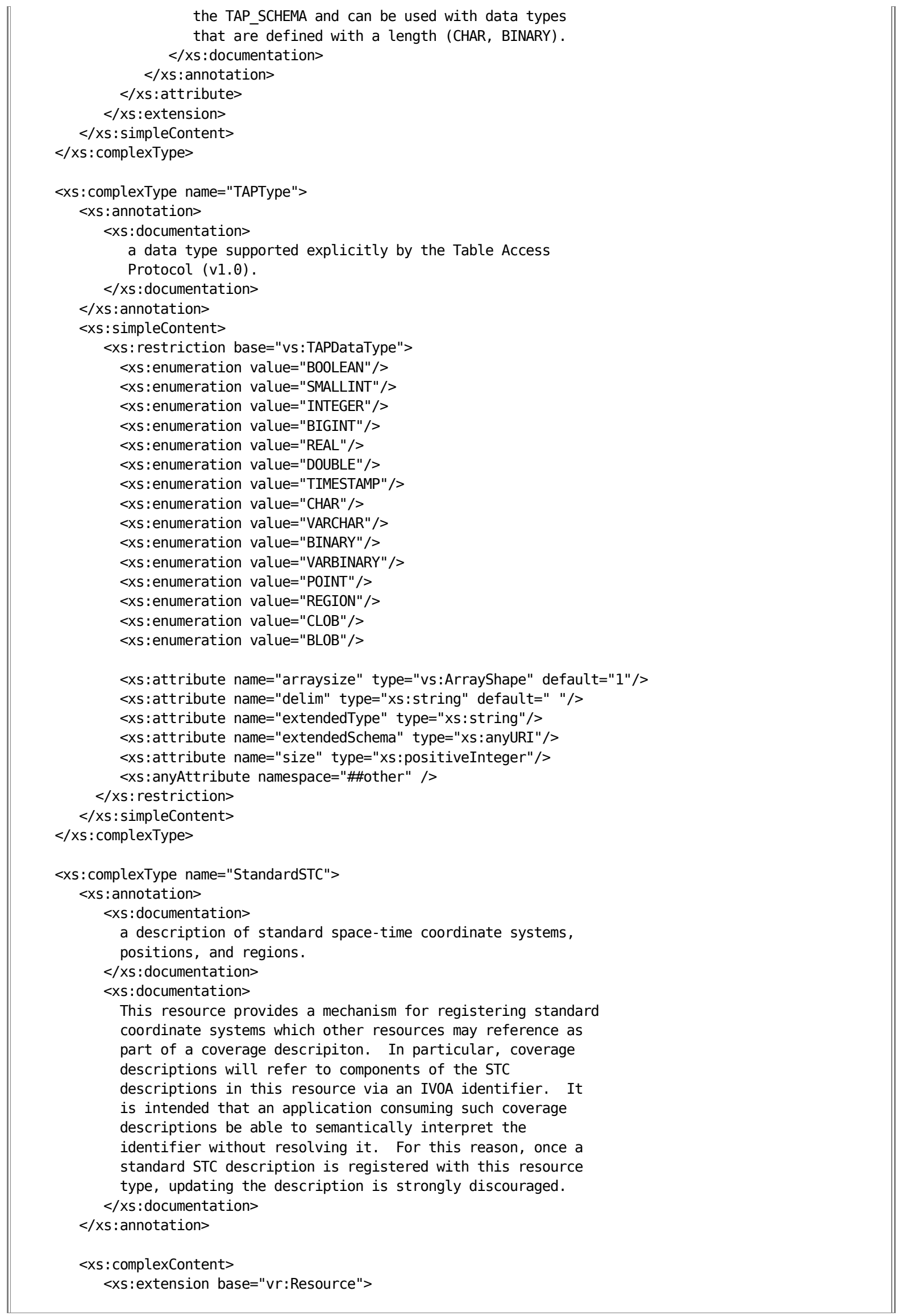




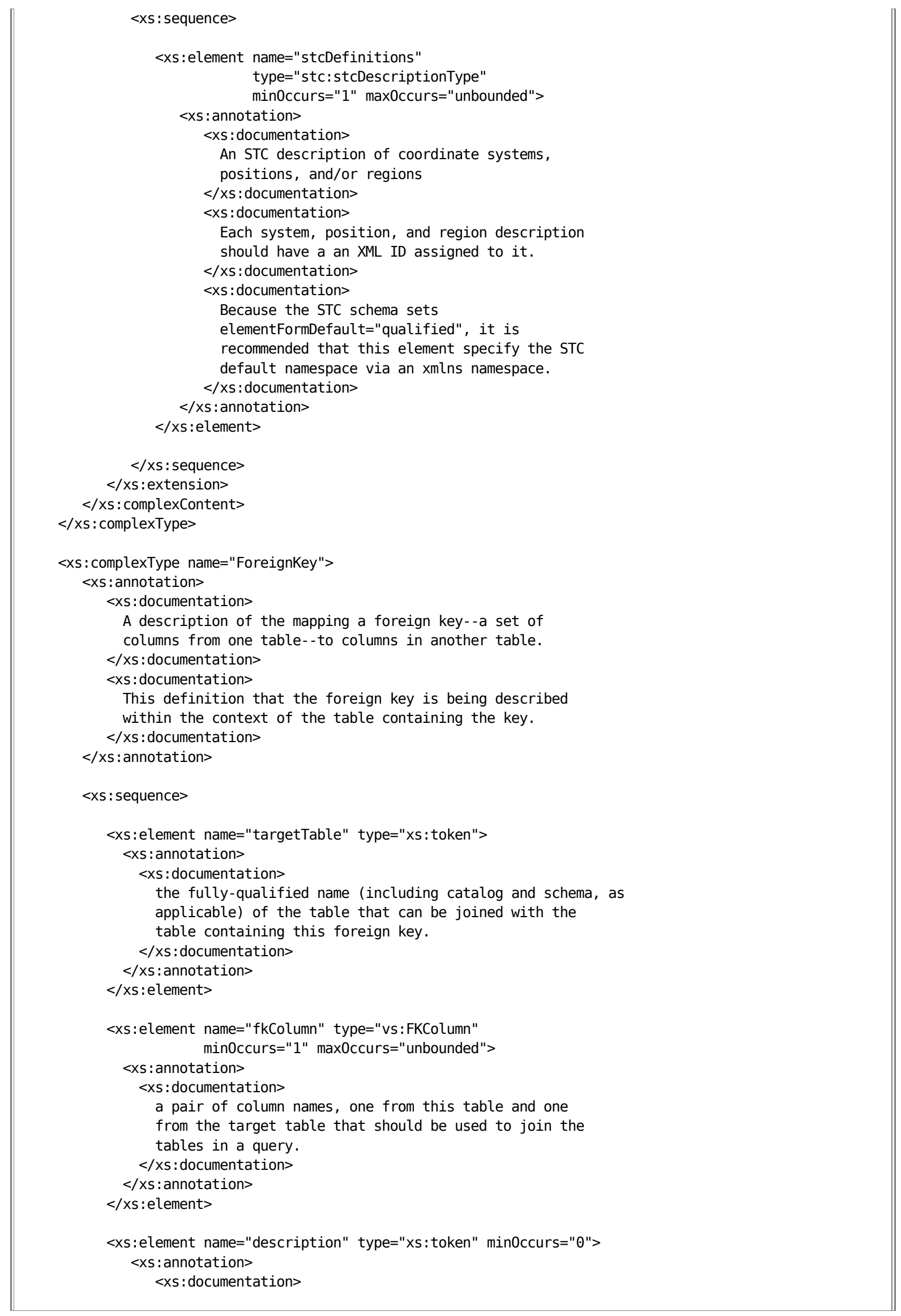




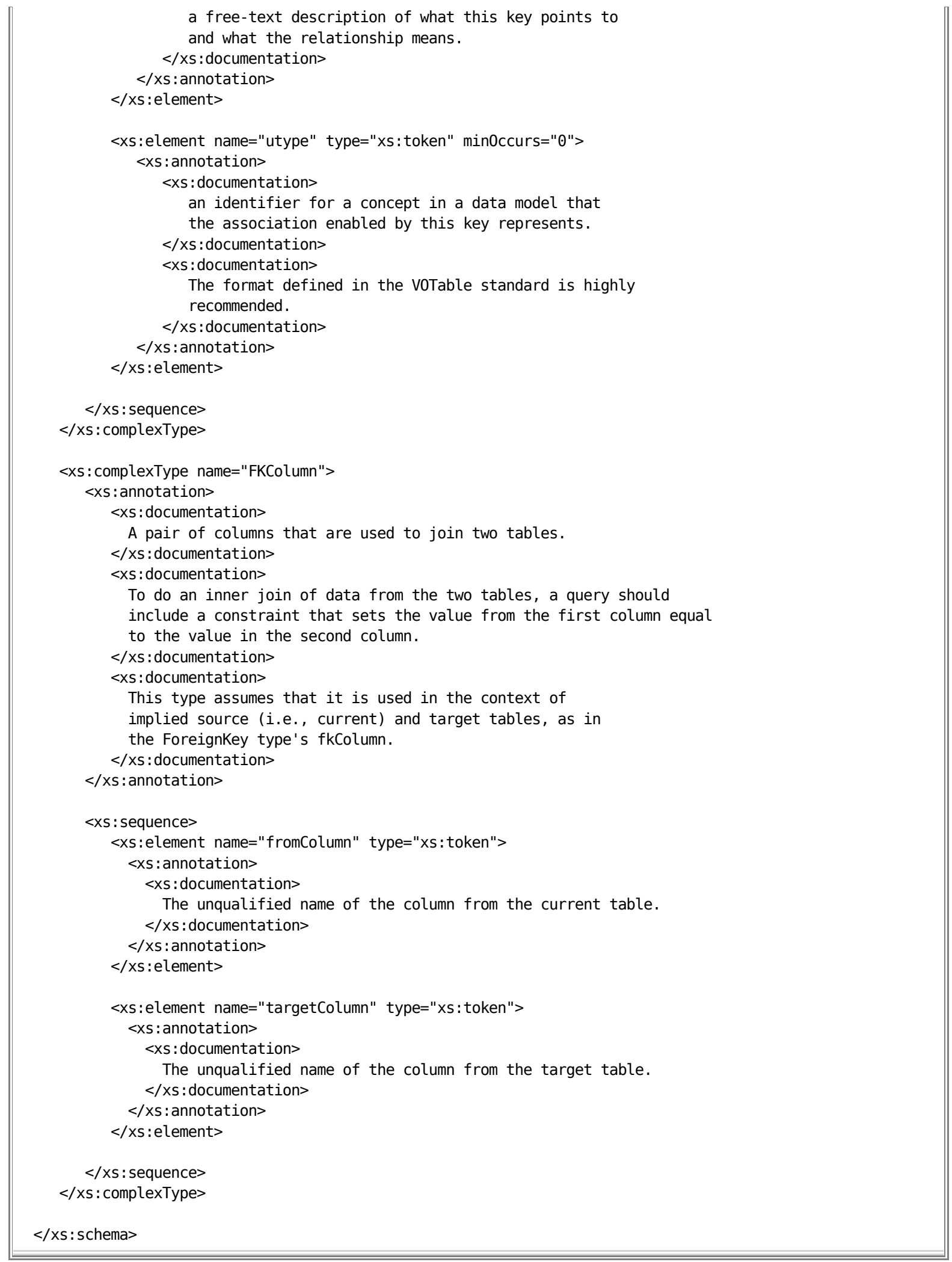

\section{Appendix B: Compatibility Issues with VODataService 1.0}

The working draft version 1.0 of the VODataService schema has been in use in IVOA 
registries since about 2008. It is expected that registries will migrate over to version 1.1 gradually and during the transition, there may well be instances of both v1.1 and v1.0 in the same registry. While the metadata structures are the mostly the same (particularly the core VOResource metadata), it is worth enumerating where they are different as this can affect how queries against differing metadata are formed.

- In v1.1, <schema> replaces v1.0's <catalog>.

- In v1.0, the root element of a table description in a vs: Datacollection was <catalogs. Consequently, a <tables element in a v1.1 record is one level lower than in v1.0.

- In v1.0, the root element of a table description in a vs: Catalogservice was <table>. Consequently, a <tables element in a v1.1 record is one level lower than in v1.0.

- Version 1.1's vs: Coverage type now contains a <regionofRegard> element. In v1.0, this metadatum was only available via coverage/stc:STCResourceProfile/stc:AstroCoord/stc:Size.

- Version 1.1 's vs: TableParam (for describing table columns) adds <utype> and <flag> elements. The v1.1 vs:InputParam adds a <utype> element.

\section{Appendix C: Change History}

\section{Changes since PR-20100916:}

- updated status for elevation to Recommendation.

- cleaned-up mis-labeled and mis-ordered change history.

\section{Changes since PR-20100914:}

- added change history for PR-20100412.

- added Note about STC mark-up in 3.2

- reworded sentence describing content of vs:DataType in section 3.5.

\section{Changes since PR-20100412:}

- fix numerous typos discovered in TCG review

- added section 1.1 to describe role of standard in the VO architecture, including diagram.

- corrected frequency range for the UV waveband

- corrected links to reference documents

\section{Changes since PR-20090903:}

- S3.4: added <: testQuery> to vs:ParamHTTP

- S3.1.1: in text, added explanation of vs:Format

- grammatical clean-up

\section{Changes since WD-20090508 (v1.10):}

- corrected errors in example in Introduction

- added <description> and <utypes elements to the vs:Foreignkey type for consistency with TAP.

- changed type names vs: TAP to vs:TAPType and vs:voTable vs:VoTableType.

\section{References}




\section{[Arch]}

Arviset, Christophe and the IVOA Tehcnical Coordination Group 2010, The IVOA in 2010: Technical Assessment and Roadmap, v1.0, IVOA Note, in preparation.

\section{[ADQL]}

Ortiz, I., Lusted, J., Dowler, P., Szalay, A., Shirasaki, Y., Nieto-Santisteban, M., Ohishi, M., O'Mullane, W., Osuna, P. 2008, IVOA Astronomical Data Query Lanquage, v2.00, IVOA Recommendation, http://wmw.ivoa.net/Documents/latest/ADQL.html

\section{[HTTP]}

Fielding, R., Gettys, J, Mogul, J., Frystyk, H., Masinter, L., Leach, P., Berners-Lee, T. 1999. Hypertext Transfer Protocol -- HTTP/1.1, IETF RFC 2616, http://wmw. ietf.org $/ \mathrm{rfc} / \mathrm{rfc2616.txt}$

\section{[RFC 2119]}

Bradner, S. 1997. Key words for use in RFCS to Indicate Requirement Levels, IETF [RM] RFC 2119, http://wnw.ietf.org/rfc/rfc2119.txt

Hanisch, Robert (ed.) 2004. Resource Metadata for the Virtual Observatory, Version 1.12, IVOA Recommendation, http://www. ivoa.net/Documents/REC/ResMetadata/RM-20040426.htm

Benson, Kevin, Plante, Ray, Auden, Elizabeth, Graham, Matthew, Greene, Gretchen, Hill, Martin, Linde, Tony, Morris, Dave, O'Mullane, Wil, Rixon, Guy, Andrews, Kona 2008, IVOA Registry Interfaces, v1.0, IVOA Recommendation, http://www. ivoa. net /Documents/RegistryInterface/20091104/

\section{[schema]}

Fallside, David C., Walmsley, Priscilla (editors) 2004, XML Schema Part 0: Primer Second Edition, W3C Recommendation 28 October 2004, http://www.w3.org

[SCS] /TR/xmlschema- 0 /

Williams, Roy, Hanisch, Robert, Szalay, Alex and Plante, Ray 2008, Simple Cone Search Version 1.03, IVOA Recommendation, http://www.ivoa. net/Documents/REC/DAL

[SIA] /ConeSearch-20080222.html.

Tody, Doug, Plante, Ray, and Harrison, Paul 2009, Simple Image Access Specification [STC]

Version 1.0, IVOA Recommendation, http://wmw.ivoa. net/Documents/SIA/20091116/.

Rots, Arnold 2007, Space-Time Coordinate Metadata for the Virtual Observatory, v1.33, IVOA Recommendation, href="http://wmw. ivoa. net/Documents/REC/STC/STC-20071030.html">

\section{[SQLGuide]}

Date, C.J. and Darwin, Hugh 1997, A Guide to the SQL Standard, Fourth Edition, (Addison[TAP] Wesley, Longman Inc.: Reading), p 24.

Dowler, P., Rixon, G., Tody, D. 2010, Table Access Protocol, v1.0, IVOA [UCD]

Recommendation, http://wnw. ivoa. net/Documents/TAP/20100327/

Martinez, A.P., Derriere, S., Delmotte, N., Gray, N., Mann, R., McGlynn, T., Ochsenbein, F., Osuna, P., Rixon, G., Williams, R. 2007, The UCD1+ Controlled Vocabulary, v1.23, IVOA Recommendation, http://wmw.ivoa. net/Documents/latest/UCDlist.html [VOR]

Plante, R., Benson, K., Graham, M., Greene, G., Harrison, P., Lemson, G., Linde, T., Rixon, G., Stébé, A. 2008, VOResource: an XML Encoding Schema for Resource Metadata, v1.03, IVOA Recommendation, http://mww.ivoa. net/Documents/REC/ReR/voResource20080222. html

[VOSI]

Graham, M. and Rixon, G. 2010, IVOA Support Interfaces, v1.00 20101129, IVOA 
Proposed Recommendation, http://Www.ivoa. net/Documents/V0SI/20100311/PR-VOSI-

1.0-20100311. html

\section{[VOTable]}

Ochsenbein, F., Williams, R., Davenhall, C., Durand, D., Fernique, P., Giaretta, D., Hanisch, R., McGlynn, T., Szalay, A., Taylor, M.B., Wicenec, A. 2008, voTable Format Definition, version 1.20, IVOA Recommendation, http://mww.ivoa.net/Documents/VoTable /20091130/

\section{[xml]}

Bray, Tim, Paoli, Jean, Sperberg-McQueen, C. M., Maler, Eve, Yergeau, Francois (editors) 2004, Extensible Markup Language (XML) 1.0 (Third Edition), W3C Recommendation 04 February 2004, http://wmw.w3.org/TR/REC-xml 